\title{
Analysis of Evolving Oil Spills in Full-Polarimetric and Hybrid-Polarity SAR
}

\author{
Martine M. Espeseth, Stine Skrunes, Member, IEEE, Cathleen. E. Jones, Member, IEEE, Camilla Brekke, \\ Member, IEEE, Benjamin Holt, Member, IEEE, and Anthony P. Doulgeris, Member, IEEE
}

\begin{abstract}
Oil spill detection using a time series of images acquired off Norway in June 2015 with the Uninhabited Aerial Vehicle Synthetic Aperture Radar (UAVSAR) is examined. The relative performance of a set of features derived from quadpolarization vs. hybrid-polarity modes in detection of various types of slicks as they evolve on a high wind driven sea surface is evaluated. It is shown that the hybrid-polarity mode is comparable to the full-polarimetric mode in its ability to distinguish the various slicks from open water for challenging conditions of high winds $(9-12 \mathrm{~m} / \mathrm{s})$, small release volumes $(0.2$ - $0.5 \mathrm{~m}^{3}$ ), and during the period $0-9$ hours following release. The features that contain the cross-polarization component are better for distinguishing the various slicks from open water at later and more developed stages. Although these features are not available in the hybrid-polarity mode, we identify alternative features to achieve similar results. In addition, a clear correlation between the results of individual features and their dependence on particular components within the two-scale Bragg scattering theory is identified. The features that show poor detectability of the oil slicks are those that are independent of the small-scale roughness, while the features resulting in good separability were dependent on several factors in the two-scale Bragg scattering model. We conclude that the hybrid-polarity mode is a viable alternative for SAR-based oil spill detection and monitoring that provides comparable results to those from quad-polarimetric SAR.
\end{abstract}

Index Terms-Synthetic Aperture Radar, Hybrid-polarity, Oil spill observation, NORSE2015, UAVSAR, Time series

\section{INTRODUCTION}

Spaceborne and airborne remote sensing instruments are key tools for an operational oil pollution monitoring program. Spaceborne instruments offer the unique capabilities of large swath widths and for some satellite constellation missions improved temporal coverage. Aircraft surveillance flights are flexible and allow monitoring of evolving oil with time, as well as verifying the oil in some cases. In recent years, characterizing oil spills in the marine environment using full-polarimetric (FP) Synthetic Aperture Radar (SAR) has intensified (see, e.g., [1]-[4]). During the Deepwater Horizon accident, the NASA Uninhabited Aerial Vehicle Synthetic Aperture Radar (UAVSAR) provided valuable observations of the major oil spill with fine resolution and a system that has a low noise

M. M. Espeseth, S. Skrunes, C. Brekke, and A. P. Doulgeris are with the Department of Physics and Technology, UiT The Arctic University of Norway, 9037 Troms $\varnothing$, Norway (e-mail: martine.espeseth@uit.no; stine.skrunes@uit.no; camilla.brekke@uit.no; anthony.p.doulgeris@uit.no).

C. E. Jones and B. Holt are with the Jet Propulsion Laboratory, California Institute of Technology, Pasadena, CA 91125 USA (e-mail: cathleen.e.jones@jpl.nasa.gov; benjamin.m.holt@jpl.nasa.gov). floor [5]. Analysis of the FP SAR acquisitions revealed a potential in retrieval of quantitative slick properties [1].

FP SAR systems provide a unique capability of measuring the complete scattering matrix and allow identification and extraction of the scattering properties within a given resolution cell. However, the FP SAR system comes at a cost, typically a smaller spatial coverage or reduced spatial resolution compared to the dual-polarization (DP) and single-polarization SAR modes. A DP SAR transmits in one polarization and receives in two polarization channels. The choice of polarization for the conventional linear-linear DP SAR systems is horizontal $(\mathrm{H})$ or vertical $(\mathrm{V})$ linear polarization on transmit, and the backscattered response is measured in the horizontal and vertical linearly polarized channels. The drawback of using a DP SAR system is the reduced polarimetric information compared to FP. In [6], Raney suggested that changing the polarization of the transmitted wave to circular polarization (resulting in a circular-circular system) gave a simpler instrument and improved the quality of the radar measurements in terms of minimizing sensitivity to relative errors and crosstalk, straight forward calibration of the radar signals, and decreasing the on-board resource requirements. This mode was named hybrid-polarity (HP) or compact-polarimetric (CP) mode. In addition, the polarimetric information given in the HP (CP) mode is in some cases reported to be close to that of FP SARs (see, e.g., [7]-[10]). The HP mode belongs in the DP SAR group, with wider swath and equal spatial resolution or improved spatial resolution and equal swath compared to the conventional FP SARs.

There exist a few studies on oil spill detection related to the use of simulated HP UAVSAR data from the Deepwater Horizon in the Gulf of Mexico from 2010 (see, e.g., [11][12]). In [11], the authors investigated the reconstruction of a pseudo FP covariance matrix from simulated HP data and computed the oil-water mixing index (Mdex) suggested in [13]. The authors in [12] investigated some simulated HP features and analyzed the appearance of the oil in the Deepwater Horizon slick using one UAVSAR scene covering a relatively thick oil slick under low wind conditions.

The backscattered response from clean seas and oil slicks within SAR scenes is complex and dependent on several factors, including amongst others the slick characteristics (dielectric properties, viscosity, extent, composition, etc.), environmental conditions (wind, sea state, temperature, etc.), and sensor properties (frequency, resolution, coverage, signal-tonoise ratio, etc.). In general, oil spills will spread to form a thin layer on the water surface, and this layer will dampen 
the small-scale roughness on the ocean surface, resulting in reduced backscattered power [14]. Another factor that can reduce the backscattered power is a reduction in the dielectric constant within the slick compared to the clean sea. This factor will contribute to the detectability if the oil slick is thick enough and/or the concentration of the oil droplets within the water column is high enough [13]. The backscattering of microwaves from a clean sea surface is usually described using Bragg scattering theory, in which the incoming wave is in resonance with the ocean waves (resonant scattering) [15] [16]. The Bragg waves can further be modulated by the longer waves on which they ride through tilt and hydrodynamic effects [17].

For the first time, in the study reported here we investigate the difference between FP and HP for a series of UAVSAR scenes covering various types of oil slicks under high wind condition as they evolve following release. This paper investigates and compares FP and simulated HP data acquired over slicks using a unique UAVSAR time series acquired in FP mode. The UAVSAR time series was collected from a controlled oil spill experiment, the NOrwegian Radar oil Spill Experiment 2015 (NORSE2015), that took place in the North Sea at the Frigg field in June 2015. This experiment was a collaboration between UiT The Arctic University of Norway, the Jet Propulsion Laboratory (JPL) / National Aeronautics and Space Administration (NASA) and the Norwegian Clean Seas Association for Operating Companies (NOFO). The UAVSAR time series was collected during two flights during a single day with approximately three hours between the end of data acquisition in the first and the beginning of data acquisition in the second. There were 16 and 6 acquisitions in the first and second flights, respectively, obtained over an eight-hour total time span. The UAVSAR was used to image four different oil slicks as they evolved and weathered on a high wind sea surface (approximately $12 \mathrm{~m} / \mathrm{s}$ ). In addition, X-, C-, and Lband SAR data were also collected from satellite SAR sensors coincident with one of the UAVSAR images. The reader is referred to [18], [19], and [20] for additional information and analysis from the experiment.

The main objectives of the work reported here are i) to study the performance of a set of well known FP and HP features to detect oil slicks, ii) identify and compare the best FP and HP features for detecting the evolving oil slicks, iii) identify the dominating components in the Bragg scattering theory to which the investigated FP and HP polarimetric features are sensitive, and iv) study the difference in detectability of the various oil slicks as they developed.

Bragg scattering theory and the HP theory is described in Section II, the experiment is described in Section III, preprocessing steps are described in Section IV and in Appendix $\mathrm{A}$, and Section $\mathrm{V}$ contains information about the polarimetric features used in the study. Section VI presents the time series results, and Section VII presents conclusions from the study.

\section{THEORY}

In this section we introduce the tilted Bragg scattering model used for the FP data, the theory of the HP SAR mode, and the two-scale Bragg model that is adapted for the HP mode.

\section{A. The tilted Bragg model}

The backscatter from the ocean surface can be described through the theory of Bragg. Bragg scattering is caused by small-scale surface roughness whose height is small compared to the radar wavelength [15]. In addition, the in-plane tilt and out-of-plane tilt of the facet, caused by the large-scale gravity waves on the ocean surface, will alter a response in the like-polarized channel and add a response in the crosspolarized channel. Including this tilt of the surface in the Bragg model leads to the tilted Bragg model (also known as the Valenzuela model) [15]. From this model the equations of the normalized radar cross sections from a FP SAR system are given in (1), (2), and (3). In these equations $k_{r}$ is the wavenumber, $\theta$ is the incidence angle relative to the untilted horizontal plane [1], and $\theta_{i}=\cos ^{-1}[\cos (\theta+\psi) \cos (\xi)]$ is the local incidence angle. $\psi$ is the angle between the vertical and the normal to the patch projected into the plane of incidence, and $\xi$ is the angle between the vertical and the normal to the patch projected into the plane perpendicular to the plane of incidence [15]. $W(\cdot)$ is the 2-D wavenumber spectral density of the ocean surface roughness, and $R_{H H}$ and $R_{V V}$ are the Bragg scattering coefficients defined as [1]:

$$
R_{H H}\left(\theta_{i}, \epsilon_{r}\right)=\frac{\cos \left(\theta_{i}\right)-\sqrt{\epsilon_{r}-\sin ^{2}\left(\theta_{i}\right)}}{\cos \left(\theta_{i}\right)+\sqrt{\epsilon_{r}-\sin ^{2}\left(\theta_{i}\right)}}
$$

and

$$
R_{V V}\left(\theta_{i}, \epsilon_{r}\right)=\frac{\left(\epsilon_{r}-1\right)\left(\sin ^{2}\left(\theta_{i}\right)-\epsilon_{r}\left(1+\sin ^{2}\left(\theta_{i}\right)\right)\right)}{\left(\epsilon_{r} \cos \left(\theta_{i}\right)+\sqrt{\epsilon_{r}-\sin ^{2}\left(\theta_{i}\right)}\right)^{2}}
$$

where the $\epsilon_{r}$ is the relative dielectric constant, and the subscripts of $R_{H H}$ and $R_{V V}$ represent the transmit and the receive polarizations. As can be observed from the above equations, the backscattered radar cross sections are dependent on several components, namely, the wave spectrum, imaging geometry and the dielectric properties of the media.

\section{B. Hybrid-polarity theory}

In this study, we investigate the HP system with right circular transmit and linear receive architecture. This configuration is already integrated in current satellite missions, such as RISAT-1 and ALOS-2, and will also be incorporated in several upcoming spaceborne SARs. Recognizing its great potential for oil spill detection identified in [10] [11], we choose HP as a comparing system to FP SAR in this work. The fundamental quantities measured by a polarimetric SAR system are the complex backscattering terms $\mathrm{S}_{i j}$. Here, $i$ and $j$ define the polarizations of the transmit and receive channels in the radar system. The HP mode transmits only one circular polarization, either left (L) or right (R), and receives two orthogonal linear polarizations, namely horizontal and vertical [6]. For the right circular HP mode, the scattering vector is defined as

$$
\bar{k}_{(R H, R V)}=\left[S_{R H}, S_{R V}\right]^{T},
$$




$$
\begin{aligned}
\sigma_{H H}^{0}= & 4 \pi k_{r}^{4} \cos ^{4}\left(\theta_{i}\right)\left|\left(\frac{\sin (\theta+\psi) \cos (\xi)}{\sin \left(\theta_{i}\right)}\right)^{2} R_{H H}\left(\theta_{i}, \epsilon_{r}\right)+\left(\frac{\sin (\xi)}{\sin \left(\theta_{i}\right)}\right)^{2} R_{V V}\left(\theta_{i}, \epsilon_{r}\right)\right|^{2} \\
& \times W\left(2 k_{r} \sin (\theta+\psi), 2 k_{r} \cos (\theta+\psi) \sin (\xi)\right) \\
\sigma_{V V}^{0} & =4 \pi k_{r}^{4} \cos ^{4}\left(\theta_{i}\right)\left|\left(\frac{\sin (\theta+\psi) \cos (\xi)}{\sin \theta_{i}}\right)^{2} R_{V V}\left(\theta_{i}, \epsilon_{r}\right)+\left(\frac{\sin (\xi)}{\sin \left(\theta_{i}\right)}\right)^{2} R_{H H}\left(\theta_{i}, \epsilon_{r}\right)\right|^{2} \\
& \times W\left(2 k_{r} \sin (\theta+\psi), 2 k_{r} \cos (\theta+\psi) \sin (\xi)\right) \\
\sigma_{H V}^{0} & =4 \pi k_{r}^{4} \cos ^{4}\left(\theta_{i}\right)\left(\frac{\sin (\theta+\psi) \cos (\xi) \sin (\xi)}{\sin ^{2}\left(\theta_{i}\right)}\right)^{2}\left|R_{V V}\left(\theta_{i}, \epsilon_{r}\right)-R_{H H}\left(\theta_{i}, \epsilon_{r}\right)\right|^{2} \\
& \times W\left(2 k_{r} \sin (\theta+\psi), 2 k_{r} \cos (\theta+\psi) \sin (\xi)\right)
\end{aligned}
$$

where $\mathrm{T}$ denotes the transpose operator. The right circularly polarized transmit mode is used throughout this study. In the linear horizontal and vertical basis the scattering vector is expressed as [21]:

$$
\bar{k}_{(R H, R V)}=\frac{1}{\sqrt{2}}\left[S_{H H}-i S_{H V},-i S_{V V}+S_{H V}\right]^{T},
$$

where reciprocity is assumed $\left(S_{H V}=S_{V H}\right)$. The UAVSAR instrument is a FP radar, and the HP scattering vector is simulated based on (7). In the FP SAR data we have both co- and cross-polarized channels. By looking at the scattering vector $\bar{k}_{(R H, R V)}$ we can observe that the co- and crosspolarized components are not possible to isolate, since the HP scattering components are a mix of co- and cross-polarized terms. Combining the two measured linear horizontal and vertical polarization values, we can also form orthogonal components in the circular-circular polarization basis as [22]:

$$
\begin{aligned}
\bar{k}_{(R R, R L)} & =\left[S_{R R}, S_{R L}\right]^{T} \\
& =\frac{1}{\sqrt{2}}\left[-S_{R H}+i S_{R V}, i S_{R H}-S_{R V}\right]^{T} \\
& =\frac{1}{2}\left[S_{V V}-S_{H H}+2 i S_{H V}, i\left(S_{H H}+S_{V V}\right)\right]^{T} .
\end{aligned}
$$

The expected sense of received circular polarization is opposite to the transmitted sense [23]. Therefore, the $S_{R R}$ becomes the cross-polarization state, while the $S_{R L}$ is the like-polarization state [23]. This corresponds to the compact-polarimetric SAR group where the antenna transmits on right circular and receives in both right- and left-hand circular. Note, our initial starting point is still a simulated HP SAR system, but the HP scattering vector is in this case projected onto the circular basis at the receiver. Polarimetric features extracted from $\bar{k}_{(R H, R V)}$ and $\bar{k}_{(R R, R L)}$ will in later sections be investigated.

The polarimetric sample covariance matrix can be calculated from the target vector for each polarimetric system. The sample FP covariance matrix is given as:

$$
C_{F P}=\frac{1}{L} \sum_{j=1}^{L} \bar{k}_{\{j,(F P)\}} \bar{k}_{\{j,(F P)\}}^{\star T},
$$

where $\star$ represents complex conjugate, $\bar{k}_{j}$ is the $j^{\text {th }}$ Lexicographic scattering vector $\bar{k}_{j}=\left[S_{H H}, S_{H V}, S_{V H}, S_{V V}\right]^{T}$, and $\mathrm{L}$ is the number of samples included in the computation of the covariance matrix (number of looks). Similarly, the sample HP covariance matrices in the circular-linear and circular-circular basis, are given as:

$$
C_{(R H, R V)}=\frac{1}{L} \sum_{j=1}^{L} \bar{k}_{\{j,(R H, R V)\}} \bar{k}_{\{j,(R H, R V)\}}^{\star T}
$$

and

$$
C_{(R R, R L)}=\frac{1}{L} \sum_{j=1}^{L} \bar{k}_{\{j,(R R, R L)\}} \bar{k}_{\{j,(R R, R L)\}}^{\star T} .
$$

Several studies have attempted to reconstruct a pseudo FP covariance matrix, i.e., transforming from $(10)$ to 99 (see e.g., [21]-[24]). To do so, it is necessary to make some assumptions about the backscattering properties. As highlighted in [25], the appropriate methodology is to directly compare the HP with the FP mode without transforming to a pseudo FP covariance matrix, thus avoiding any assumptions. In this study, we follow Raney's methodology [25] and perform the study on features extracted directly from the simulated HP data. It is important to be aware that the use of HP mode in the UAVSAR instrument will not increase the swath width due to the design of the system. Also, when simulating the HP data from the FP data, a $3 \mathrm{~dB}$ power loss is introduced due to the $\sqrt{2}$ in 77 [8].

Only the radar cross section of the $\mathrm{HH}, \mathrm{HV}$, and VV channels are estimated in the tilted Bragg model discussed in Section III-A In order to have model estimates of the HP data, another model containing the complex backscattering coefficients is needed. In addition, a similar model is also necessary when evaluating polarimetric features from the FP covariance matrix. Therefore, in [10], the authors suggested to use the two-scale Bragg for the HP mode. This model is similar to the tilted Bragg model in terms of containing two scales of the surface roughness, namely the small- and the large-scale roughness. The authors of [10] used the two-scale model by first simulating the HP scattering vector followed by a projection to the circular basis. The matrix $(\boldsymbol{S})$ of scattering coefficients denotes the Sinclair scattering matrix. Following the two-scale model, $S$ can be expressed as [10] [26]:

$$
\boldsymbol{S}=a_{s} \boldsymbol{R}(\phi)\left[\begin{array}{cc}
R_{H H}\left(\theta_{i}, \epsilon_{r}\right) & 0 \\
0 & R_{V V}\left(\theta_{i}, \epsilon_{r}\right)
\end{array}\right] \boldsymbol{R}^{T}(\phi),
$$


where $\left|a_{s}\right|^{2}=k_{r}^{4} \cos ^{4}\left(\theta_{i}\right) W(\cdot)$ contains factors related to the small-scale roughness like the ocean wave spectrum, incidence angle, and wave number. The rotation matrix $\boldsymbol{R}(\phi)$ is given as:

$$
\boldsymbol{R}(\phi)=\left[\begin{array}{cc}
\cos (\phi) & \sin (\phi) \\
-\sin (\phi) & \cos (\phi)
\end{array}\right] .
$$

The tilt of the facet causes a rotation of the local plane of incidence around the look direction by an angle $\phi$ [27]. $\boldsymbol{R}(\phi)$ depends on the surface slope (azimuth and range directions) or large-scale roughness and the radar look angle [22] [27]. Calculating the expression in equation (12) gives (14). Inserting (14) in (7) gives (15). Both $S_{R H}$ and $S_{R V}$ depend on the rotation angle $(\phi)$, so the authors in [10] suggested to consider the following quantities:

$$
\begin{aligned}
S_{R H}-i S_{R V} & =\frac{1}{\sqrt{2}}\left(S_{H H}-S_{V V}-2 i S_{H V}\right) \\
& =\frac{a_{s}}{\sqrt{2}}\left(R_{H H}\left(\theta_{i}, \epsilon_{r}\right)-R_{V V}\left(\theta_{i}, \epsilon_{r}\right)\right) \exp (2 \phi i) \\
S_{R H}+i S_{R V} & =\frac{1}{\sqrt{2}}\left(S_{H H}+S_{V V}\right) \\
& =\frac{a_{s}}{\sqrt{2}}\left(R_{H H}\left(\theta_{i}, \epsilon_{r}\right)+R_{V V}\left(\theta_{i}, \epsilon_{r}\right)\right) .
\end{aligned}
$$

Note the similarity to equation $(8)$, where the

$$
\begin{aligned}
\bar{k}_{(R R, R L)} & =\left[\begin{array}{c}
S_{R R} \\
S_{R L}
\end{array}\right]=\frac{1}{\sqrt{2}}\left[\begin{array}{c}
-\left(S_{R H}-i S_{R V}\right) \\
i\left(S_{R H}+i S_{R V}\right)
\end{array}\right] \\
& =\frac{a_{s}}{2}\left[\begin{array}{c}
\left(R_{V V}\left(\theta_{i}, \epsilon_{r}\right)-R_{H H}\left(\theta_{i}, \epsilon_{r}\right)\right) \exp (2 \phi i) \\
i\left(R_{H H}\left(\theta_{i}, \epsilon_{r}\right)+R_{V V}\left(\theta_{i}, \epsilon_{r}\right)\right)
\end{array}\right] .
\end{aligned}
$$

The intensity of $S_{R R}$ and $S_{R L}$ becomes:

$$
\begin{aligned}
\left|S_{R R}\right|^{2} & =\left|-\frac{1}{\sqrt{2}}\left(S_{R H}-i S_{R V}\right)\right|^{2} \\
& =\frac{\left|a_{s}\right|^{2}}{4}\left|R_{V V}\left(\theta_{i}, \epsilon_{r}\right)-R_{H H}\left(\theta_{i}, \epsilon_{r}\right)\right|^{2}
\end{aligned}
$$

and

$$
\begin{aligned}
\left|S_{R L}\right|^{2} & =\left|\frac{i}{\sqrt{2}}\left(S_{R H}+i S_{R V}\right)\right|^{2} \\
& =\frac{\left|a_{s}\right|^{2}}{4}\left|R_{H H}\left(\theta_{i}, \epsilon_{r}\right)+R_{V V}\left(\theta_{i}, \epsilon_{r}\right)\right|^{2} .
\end{aligned}
$$

We observe that $S_{R L}$ is independent of the rotation angle, and so are $\left|S_{R R}\right|^{2}$ and $\left|S_{R L}\right|^{2}$. Note, however, that all of these include the Bragg coefficients, which are dependent on the tilt angles. The theory of tilted Bragg and the two-scale Bragg models will be used as a fundamental theory both when presenting the polarimetric features investigated and when evaluating the results from the UAVSAR data (sections $\mathrm{V}$ and VI.

\section{NORSE2015 EXPERIMENT}

An extensive SAR dataset was collected from both airand spaceborne platforms during the NORSE2015 experiment at the abandoned Frigg field in the North Sea. Insitu data including wind, temperature, and oil-to-water ratios of the released oils were collected and have been described in detail previously [18] [19]. The main motivation behind the NORSE2015 experiment was to collect multisensor and multifrequency SAR data to study the polarization-dependent electromagnetic signals and their relationship to varying oilwater mixtures and dielectric properties, as well as study the evolving oil slicks' drift and characteristics as a function of time using SAR [18] [19] [20] [28] [29]. Table I] summarizes information about the released oil, which were three emulsions based on the same crude oil but with different volumetric oil concentration, i.e., $40 \%$ oil (E40), 60\% oil (E60), 80\% oil (E80), and release of plant oil (PO) for simulation of a natural biogenic slick.

The VV-intensity images for the UAVSAR scenes are displayed in Fig. 1. These intensity images are geocoded, smoothed, and scaled for displaying purposes, and the ships are masked out and colored red. Note that the true width (number of pixels in the subsection displayed) of the images varies across the time series as the oil slicks evolve and spread out. The release of the E80 slick was not complete in the first UAVSAR acquisition so this scene is not used in the analysis of the E80 slick. The plant oil and the three emulsion slicks are indicated in Fig. 1, with the PO as the southern-most slick, and E40, E60, and E80 to the north. The properties of the UAVSAR sensor are given in Table II

Table I: Properties of the experimental oil releases during NORSE2015 [18] [19].

\begin{tabular}{|l|c|l|c|}
\hline Release & $\begin{array}{l}\text { Time } \\
\text { (UTC) }\end{array}$ & Substance & Volume \\
\hline PO & $04: 48$ & Plant Oil: Radiagreen ebo & $0.2 \mathrm{~m}^{3}$ \\
\hline E40 & $04: 59$ & $\begin{array}{l}\text { Emulsion (40\% oil) } \\
\text { 300 L water + 100 L Troll + } \\
100 \text { L Oseberg + 0.2 L One-Mul }\end{array}$ & $0.5 \mathrm{~m}^{3}$ \\
\hline E60 & $05: 15$ & $\begin{array}{l}\text { Emulsion } 60 \% \text { oil) } \\
\text { 200 L water + 150 L Troll } \\
150 \text { L Oseberg + 0.2 L One-Mul }\end{array}$ & $0.5 \mathrm{~m}^{3}$ \\
& & $\begin{array}{l}\text { Emulsion (80\% oil) } \\
100 \text { L water }+200 \text { L Troll }+\end{array}$ & $0.5 \mathrm{~m}^{3}$ \\
& 200 L Oseberg + 0.2 L One-Mul & \\
\hline E80 & $05: 30$ & \\
\hline
\end{tabular}

The oils were released along a line approximately parallel to the spaceborne SAR flight directions to obtain similar incidence angles for all slicks. In order to maximize the signalto-noise ratios, the releases were done close to the center of the swaths. The UAVSAR acquisitions were then adapted to this setup and the research team onboard the aircraft selected the flight lines so that the oil slicks were located where the antenna gain was near its maxima. The noise floor as a function of incidence angle (along range direction) is shown for the UAVSAR instrument in [5, Fig. 1]. Here, the minimum noise is found near mid-swath in the range direction. Fig. 2 shows the incidence angle span for each slick along the UAVSAR time series. The UAVSAR monitored the evolving slicks in three different look directions and five different imaging geometries. In order to limit the effect from the imaging geometry on the polarimetric features, only ID numbers 00709 (ascending) and 18709 (descending) are used in this study, with white background in Fig. 2. This is because these two datasets of imaging geometries contain the most scenes and the oil slicks 


$$
\begin{gathered}
\boldsymbol{S}=\left[\begin{array}{ll}
S_{H H} & S_{H V} \\
S_{H V} & S_{V V}
\end{array}\right]=a_{s}\left[\begin{array}{cc}
R_{H H}\left(\theta_{i}, \epsilon_{r}\right) \cos ^{2}(\phi)+R_{V V}\left(\theta_{i}, \epsilon_{r}\right) \sin ^{2} \phi & \cos (\phi) \sin (\phi)\left(R_{V V}\left(\theta_{i}, \epsilon_{r}\right)-R_{H H}\left(\theta_{i}, \epsilon_{r}\right)\right) \\
\cos (\phi) \sin (\phi)\left(R_{V V}\left(\theta_{i}, \epsilon_{r}\right)-R_{H H}\left(\theta_{i}, \epsilon_{r}\right)\right) & R_{V V}\left(\theta_{i}, \epsilon_{r}\right) \cos ^{2}(\phi)+R_{H H}\left(\theta_{i}, \epsilon_{r}\right) \sin ^{2} \phi
\end{array}\right](14) \\
\bar{k}_{(R H, R V)}=\left[\begin{array}{l}
S_{R H} \\
S_{R V}
\end{array}\right]=\frac{a_{s}}{\sqrt{2}}\left[\begin{array}{c}
R_{H H}\left(\theta_{i}, \epsilon_{r}\right) \cos ^{2}(\phi)+R_{V V}\left(\theta_{i}, \epsilon_{r}\right) \sin ^{2}(\phi)+i\left(\cos (\phi) \sin (\phi)\left(R_{H H}\left(\theta_{i}, \epsilon_{r}\right)-R_{V V}\left(\theta_{i}, \epsilon_{r}\right)\right)\right) \\
\cos (\phi) \sin (\phi)\left(R_{V V}\left(\theta_{i}, \epsilon_{r}\right)-R_{H H}\left(\theta_{i}, \epsilon_{r}\right)\right)-i\left(R_{H H}\left(\theta_{i}, \epsilon_{r}\right) \sin ^{2}(\phi)+R_{V V}\left(\theta_{i}, \epsilon_{r}\right) \cos ^{2} \phi\right)
\end{array}\right]
\end{gathered}
$$

Table II: Properties of the UAVSAR sensor [5] and the UAVSAR single-look complex data products for the acquired time series of images.

\begin{tabular}{|l|l|l|l|l|l|l|l|l|l|}
\hline Date & $\begin{array}{l}\text { Time } \\
(\mathrm{UTC})\end{array}$ & Mode & Polarization & $\begin{array}{l}\text { Frequency } \\
{[\mathrm{GHz}]}\end{array}$ & $\begin{array}{l}\text { Incidence } \\
\text { angle }\end{array}$ & $\begin{array}{l}\text { NESZ } \\
{[\mathrm{dB}]}\end{array}$ & $\begin{array}{l}\text { Resolution } \\
\left(\mathrm{rg}^{b} \times \mathrm{az}^{c}\right)\end{array}$ & $\begin{array}{l}\text { Swath width } \\
\text { direction }\end{array}$ \\
\hline $\begin{array}{l}10^{\text {th }} \text { June } \\
2015\end{array}$ & $\begin{array}{l}05: 32- \\
13: 18\end{array}$ & PolSAR & $\begin{array}{l}\text { Full-pol } \\
(\mathrm{HH}, \mathrm{HV}, \mathrm{VH}, \mathrm{VV})\end{array}$ & $\begin{array}{l}\text { L-band } \\
(1.26)\end{array}$ & $19.5^{\circ}$ to $67.5^{\circ}$ & $\sim-48$ to -33 & $2.5 \mathrm{~m} \times 1 \mathrm{~m}$ & $20 \mathrm{~km}$ & Left \\
\hline
\end{tabular}

${ }^{a}$ Time when starting the acquisition to the end of the acquisition (including both flight 1 and 2 ), ${ }^{b} \mathrm{rg}$ : range, ${ }^{c}$ az: azimuth

are located at approximately the same incidence angles across the two subsets. The gray background denotes the scenes that are left out of this analysis and are the ones with slightly different imaging geometry. However, these scenes will be included in a future study that analysis the effects from the imaging geometry on several polarimetric features.

\section{PREPROCESSING}

In this section we introduce the separability measure that is used when evaluating the performance in the preprocessing steps and the different polarimetric features. Further, we discuss each of the steps that are performed on the UAVSAR data prior to the polarimetric analysis.

\section{A. Separability measure}

In this study, several polarimetric features are compared in terms of how well they separate the various oil slicks from each other as well as from open water. In the literature, several statistical metrics are described that can be used to accomplish this task. We want to use a statistical distance measure that can be applied across the UAVSAR time series for the range of polarimetric features investigated. The Bhattacharyya distance in the closed-form expression [30] has been used in [10] for evaluating the separability between various oil slicks and open water using some selected polarimetric features. Similarly, the normalized distance between the means was used in [31]. Evaluating the mean and standard deviations for each polarimetric feature relative to each other has been done in several studies (see, e.g., [3] [32]). In our case, we choose to use a distance measure that captures both the mean and standard deviation of the polarimetric feature, and at the same time is defined on an interval with discrete boundaries. We use the closed-form expression of the Bhattacharyya distance and assume Gaussian distributed data. This distance measure is defined as [30]:

$$
\begin{aligned}
d_{i j} & =\frac{1}{4}\left(\mu_{j}-\mu_{i}\right)^{T}\left(\Sigma_{i}^{-1}+\Sigma_{j}^{-1}\right)\left(\mu_{j}-\mu_{i}\right) \\
& +\frac{1}{2} \log \left(\frac{\left|\Sigma_{i}+\Sigma_{j}\right|}{2 \sqrt{\left|\Sigma_{i} \Sigma_{j}\right|}}\right),
\end{aligned}
$$

where $\mu_{i}$ and $\mu_{j}$ are the mean values and $\Sigma_{i}$ and $\Sigma_{j}$ denote the covariance matrices of the classes $i$ and $j$, respectively. Superscript $T$ denotes the transpose operator. In this study, we apply this measure to each of the one-dimensional polarimetric features, i.e., $\Sigma_{i}$ is the marginal variance and the transpose operator is not necessary. This distance spans from 0 (high similarity) to infinity (low similarity). To obtain a distance measure with discrete boundaries we apply the Jeffries-Matusita (JM) distance, which takes values in the interval 0 (high similarity) to 2 (low similarity) [33]. The JM distance is defined as [33][34]:

$$
\mathbf{J M}_{i j}=2\left(1-e^{-d_{i j}}\right),
$$

where $d_{i j}$ is the Bhattacharyya distance given in 20 . The $\mathrm{JM}_{i j}$ distance is well described in [33], and has been used for sea ice classification in SAR data [35]. This measure is a function of the mean and standard deviation between feature values representing two given classes, in our case the various oil slicks and open water. The authors in [35] defined a JM of $\geq 1$ to indicate that two classes are considered to be separable. Fig. 3 shows examples on the sensitivity of the JM distance, where histograms of four slicks and open water regions and their corresponding intensity images are displayed. We note that the boundary around the edges of the slick is partially composed of both oil and water, and the pixels will therefore be a mix of these. In two of the examples in Fig. 3 the JM distance is slightly above 0.8 . These slicks are visible from the surrounding clean sea, and we therefore define JM values to be "acceptable" at a threshold of 0.8 and "confident" at 1 .

\section{B. Speckle filtering}

The backscattered signals from the surface can interfere constructively or destructively to produce bright and dark pixels in the SAR scene, known as speckle variation. Prior to calculating the polarimetric features used in this study, speckle filtering is performed using a box-car filter. Following the selection of the filter we select the window size (also known as number of looks). The choice of number of looks has a great impact on the spatial resolution and on the contrast between the oil slicks and the clean sea. With the high resolution of the UAVSAR scenes, we can afford to have a coarser resolution 


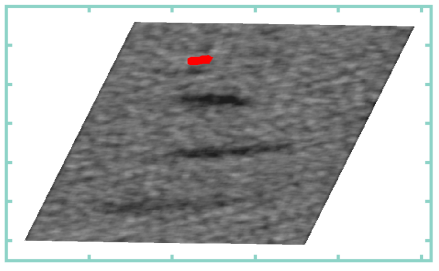

05:32 (UTC)

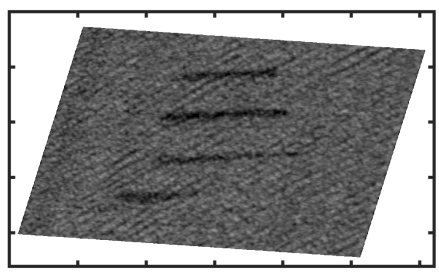

06:39 (UTC)

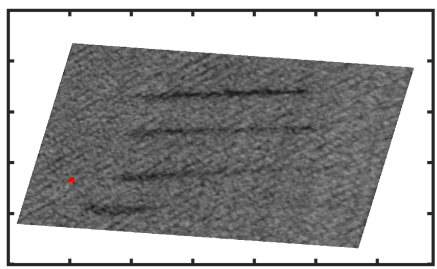

07:31 (UTC)

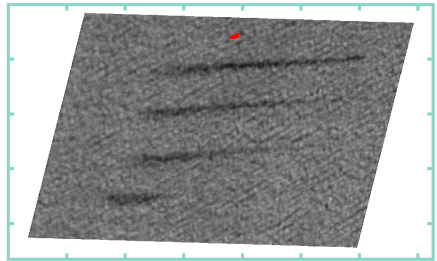

08:11 (UTC)

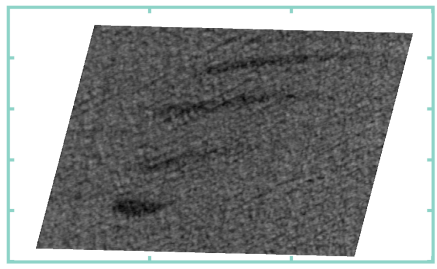

11:45 (UTC)

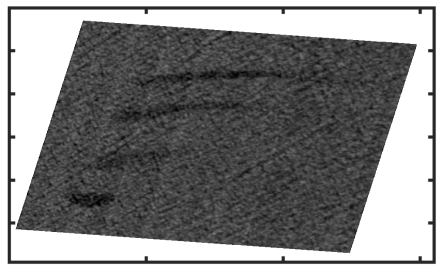

12:29 (UTC)
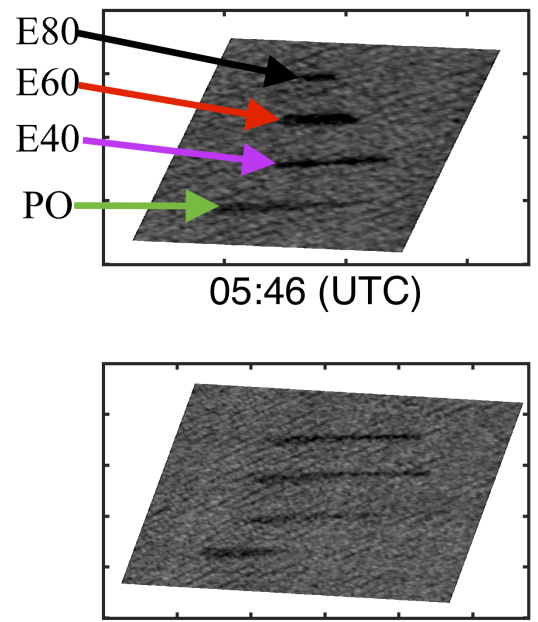

07:05 (UTC)

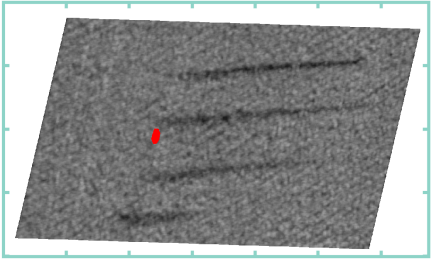

07:44 (UTC)

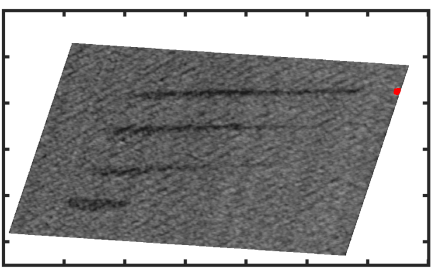

08:24 (UTC)

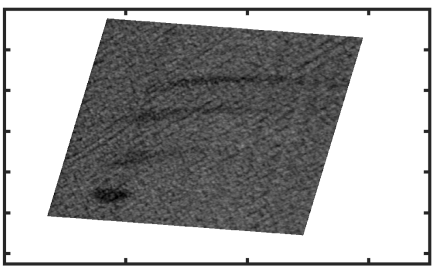

12:00 (UTC)

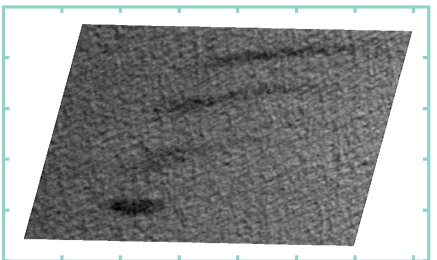

13:03 (UTC)

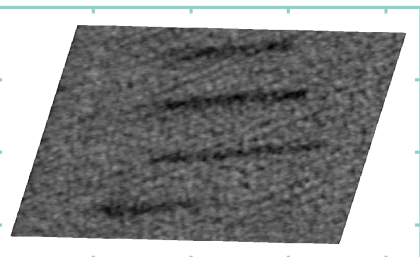

06:26 (UTC)

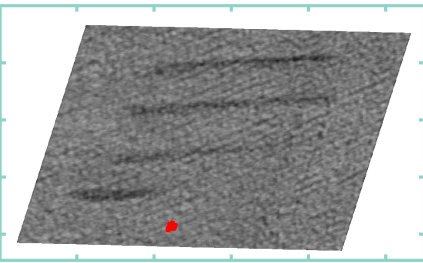

07:17 (UTC)

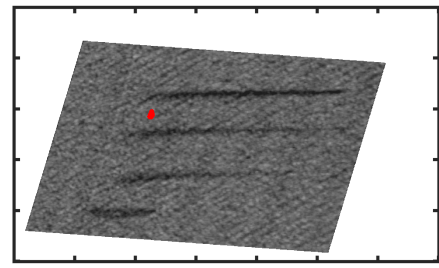

07:57 (UTC)

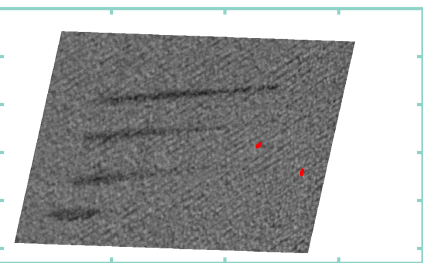

08:37 (UTC)

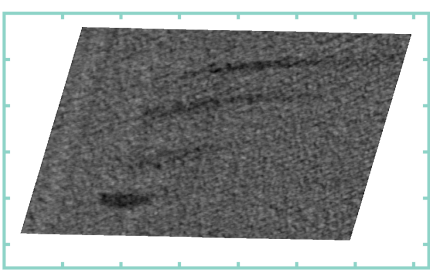

12:14 (UTC)

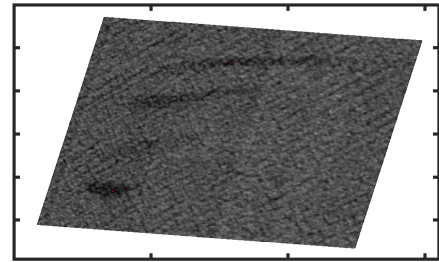

13:18 (UTC)

Figure 1: Geocoded VV-intensity for the ascending (blue box) and descending (black box) UAVSAR scenes. The images are oriented with north pointing upwards, and the ships are masked out and colored red. UAVSAR data is courtesy of NASA/JPLCaltech. 


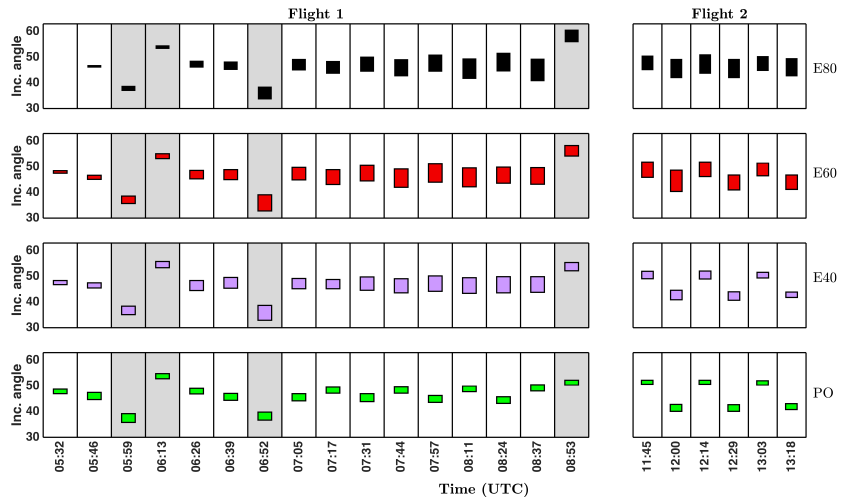

Figure 2: Overview of the incidence angle range for each slick along the UAVSAR time series. The white colored sections (a total of 18 UAVSAR scenes) are used in this analysis, and the grey colored sections are not included.

with the gain of reducing speckle. The UAVSAR ground range resolution is $2.5 \mathrm{~m}$ (slant range resolution is $1.7 \mathrm{~m}$ ) and the azimuth resolution is $1 \mathrm{~m}$ [1] [5]. In the multilooking process, the authors in [1] chose the relation to be one to four between the looks in range and azimuth direction. We apply the same relation in this analysis when multilooking the data. Additionally, to achieve a good compromise between speckle reduction and preservation of details, a sliding window is used in the feature computations.

The single look complex (SLC) images are smoothed with mask of $15 \times 60$ pixels (range $\times$ azimuth). Fig. 4 demonstrates the effect of smoothing on the JM separability of the VVdamping ratio between open water and between the four slicks as the total number of looks (both in range and azimuth) increases. The effect of increasing the number of looks in the averaging process is significant for all the JM distances of the four slicks and open water. In this case, we show the effect on two scenes, but similar results have been obtained for the other scenes as well. It is already known that increasing the mask size will enhance interpretability [36], but the small oil slicks might hamper detectability. From Fig. 4, the minimum mask size for separating the four slicks from the open water varies depending on the oil type. For these two scenes, less averaging is necessary to separate the plant oil from the open water compared to separating the emulsion slicks from the open water regions. Less averaging is needed for the E80 followed by E60 and E40. Because our main goal is to study the evolution of different oil slicks, it is important to enhance interpretability of the oil slicks and at the same time keep a high spatial resolution.

\section{Segmentation}

Our goal is to evaluate the detectability of several polarimetric features for the various slick types over the UAVSAR time series. To be able to compare detectability we need to segment out the different slicks. The same segmentation method should be applied on each scene for consistency and to avoid errors introduced by manual selection. Several segmentation methods for oil spill detection have already been extensively studied in the literature (see, e.g., [37] [38]). In our case, we choose a method that is generic and relatively simple to use, namely the "extended polarimetric feature space" (EPFS) unsupervised method described in [39] and [40]. This unsupervised segmentation method includes both polarimetric and textural information from the SAR data, and groups all pixels with similar statistical properties in the same clusters.

The intensity variation related to incidence angle can be larger than the intensity difference between the classes, and hence the oil slicks might be neglected in the original segmentation. Furthermore, the oil slicks spread out in the range direction with time, increasing the incidence angle span across the slicks. Therefore, an incidence angle correction (described in Appendix A is applied on the scattering vector prior to segmentation.

The EPFS method can be split into four stages. The first is extraction of input features from the SAR data. Here, we use the span and the relative kurtosis (see [40]) as input. This stage also includes transforming the extracted features to partially remove non-Gaussian spreading and improve symmetry of the clusters, which is often achieved with the log operator. The second stage is to sub-sample the input features to speed up the segmentation process. In the third stage, the clusters are created using the expectation maximization (EM) algorithm, assuming a multivariate Gaussian model for the transformed features. The number of classes (clusters) is usually a necessary input choice when segmenting, but this approach instead automatically determines the number of clusters using a goodness-of-fit test stage, and sequentially applies the EMalgorithm. Finally, a discrete Markov random field (MRF) contextual smoothing stage completes the segmentation by integrating contextual information to improve the connectivity within the image segments. After the unsupervised segmentation approach, the oil slick regions are manually chosen from the output segments and labeled based on the in-situ data.

Fig. 5 illustrates the segmentation results for the different slicks in the UAVSAR acquisition at 06:26 UTC. The green mask is plant oil, pink is E40, red is E60, and black is E80. Several open water regions are selected to determine the variance in the clean water properties, and to enable a reasonable representation of the polarimetric features representing the open water class under the same environmental conditions as the slicks. These are selected based on the same shape for each slick as seen in Fig. 5 This is done in order to have an equal number of open water samples as the oil slick as well as an equal number of pixels in both range and azimuth direction, which matches the incidence angles of the slick pixels.

\section{POLARIMETRIC FEATURES FOR SLICK OBSERVATION}

There exists several studies of the performance of oil slick characterization based upon various polarimetric features extracted from FP and linear-linear DP SAR data evaluated for different ocean and wind conditions, various oil types, and different sensors with various incidence angles and frequencies, see e.g., [1] [3] [32]. The sensitivity of polarimetric features to the different factors varies. This section presents the most frequently evaluated polarimetric features extracted 

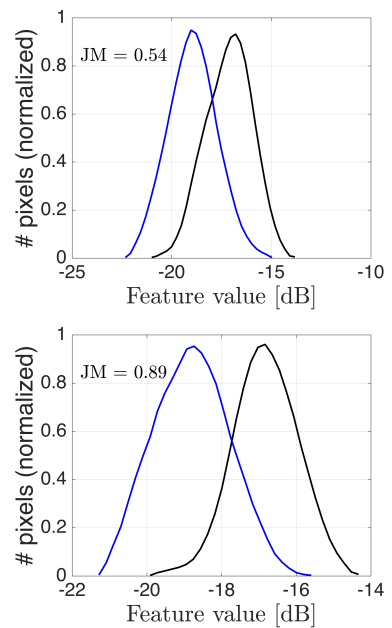
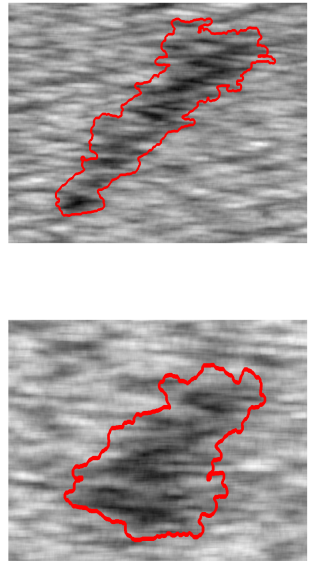
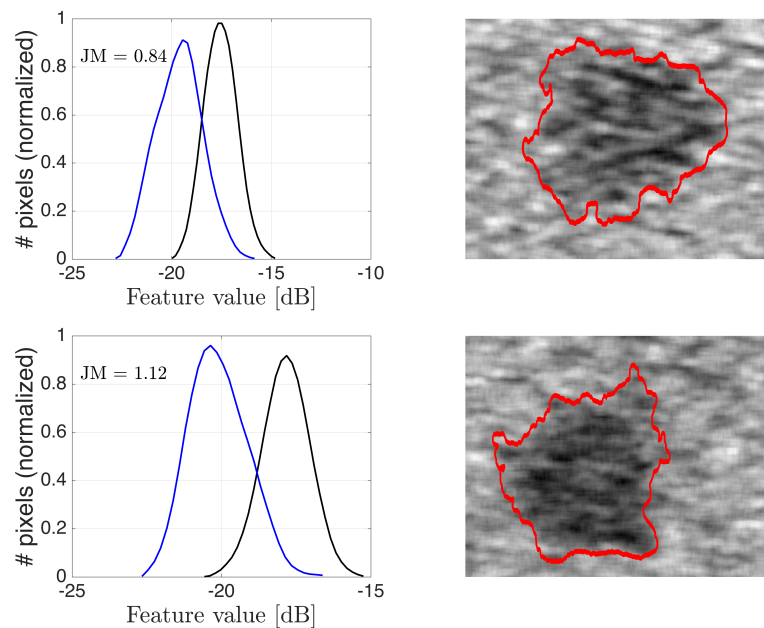

Figure 3: The sensitivity of various values of the JM distance using the VV-intensity is illustrated using histograms of the VV-intensity of four oil slicks (black color) and open water (blue color) with the same incidence angle range. The JM distance is calculated between the oil slick region and an open water region. Intensity images with the outline of the segmented masks are given to the right of their corresponding histograms.
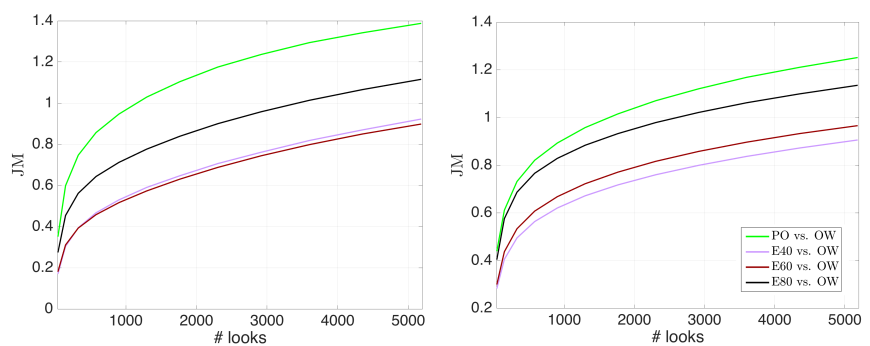

Figure 4: Effect of increasing the number of looks in the speckle filtering on the JM separability between the four slicks and open water using the VV-damping ratio. Left figure is the UAVSAR acquisition taken at 07:17 UTC and the right figure is the scene acquired at 07:44 UTC.

from FP and HP SAR data based on previous studies. A rigorous analysis is presented, connecting the Bragg scattering theory discussed in Section $\Pi$ and the polarimetric features here investigated (from both the FP and simulated HP mode).

\section{A. Full-polarimetric features}

The FP features used in this analysis are given in Table III and their corresponding relation to the components of the Bragg scattering theory is shown in Table IV] Table IV shows all the polarimetric features investigated (both FP and HP) and their dependency on factors in the Bragg scattering theory discussed in Section III The FP features have all been extensively tested for oil spill characterization and detection, and some corresponding references for these studies are given in parentheses in Table III In this study we observe oil slicks with different chemical and physical properties under high wind conditions and with relatively small volumes of slick material. Hence, some of the FP features reported as having the best performance in the literature may not fulfill their potential in our case, as already highlighted in [19]. Using one of the

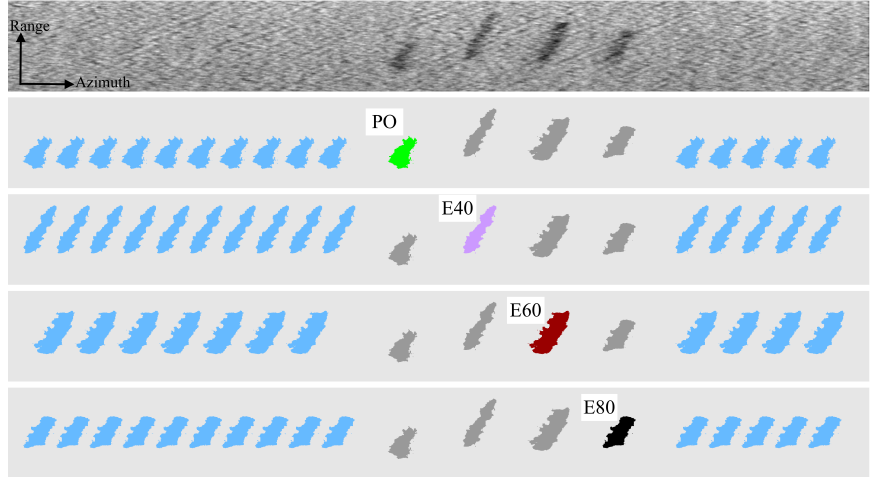

Figure 5: The top-most figure is the VV-damping ratio of the UAVSAR acquisition taken at 06:26 UTC. The figures below show the segmentation results for each of the four slicks, and the manually selected open water regions to which they are compared. Multiple water regions are used to determine the variance in the clean water properties. The green color represents the plant oil, and the pink, red, and black represent E40, E60, and E80, respectively. Blue represents open water regions.

UAVSAR acquisitions, the authors of [19] showed that the best features for separating the various oil slicks from the open water region were the VV-intensity, the geometric intensity, the largest eigenvalue of the polarimetric decomposition, the real part of the copolarization cross product, and the span (as defined in Table III). These features had the highest separability (Fisher discriminant ratio) between the four slicks and open water.

The damping ratios have been shown to be good features for evaluating the contrast between the slick-free and slick covered surfaces in SAR imagery, see, e.g., [1] [3] [41] [42]. Both measured and simulated damping ratios are reported to decrease 
Table III: Overview of the investigated FP polarimetric features (computed using a mask of $15 \times 60$ pixels). The references included are examples of studies where the features have been studied for oil spill observation.

\begin{tabular}{|c|c|}
\hline \multicolumn{2}{|c|}{ FP Features } \\
\hline Name & Formula \\
\hline Damping ratio ([1] 3$][41,42])$ & $\begin{array}{l}\zeta_{i j}=10 \log _{10}\left(\frac{\left\langle I_{i j}^{(\text {sea })}\right\rangle}{\left\langle I_{i j}^{(\text {slick })}\right\rangle}\right), I_{i j}=\left|S_{i j}\right|^{2} \\
(i, j) \in\{(\mathrm{H}, \mathrm{H}) \vee(\mathrm{H}, \mathrm{V}) \vee(\mathrm{V}, \mathrm{H}) \vee(\mathrm{V}, \mathrm{V})\}\end{array}$ \\
\hline Copolarization power ratio $([1][2])$ & $\gamma_{C O}=\frac{\left\langle I_{V V}\right\rangle}{\left\langle I_{H H}\right\rangle}$ \\
\hline $\begin{array}{l}\text { Real and imaginary part of the copolarization } \\
\text { cross product }([2][3][19])\end{array}$ & $r_{C O}=\left|\Re\left(\left\langle S_{H H} S_{V V}^{\star}\right\rangle\right)\right|, i_{C O}=\left|\Im\left(\left\langle S_{H H} S_{V V}^{\star}\right\rangle\right)\right|$ \\
\hline $\begin{array}{l}\text { Standard deviation of the copolarization } \\
\text { phase difference }([4][43])\end{array}$ & $\phi_{C O}=\sqrt{\left\langle\left(\phi_{H H}-\phi_{V V}\right)^{2}\right\rangle+\left(\left\langle\phi_{H H}-\phi_{V V}\right\rangle\right)^{2}}$ \\
\hline $\begin{array}{l}\text { Magnitude of the copolarization correlation } \\
\text { coefficient }([2][9][44][45][44 \mid)\end{array}$ & $\rho_{C O}=\frac{\left|\left(S_{H H} S_{V V}^{\star}\right)\right|}{\sqrt{\left\langle\left|S_{H H}\right|^{2}\right\rangle\left\langle\left|S_{V V}\right|^{2}\right\rangle}}$ \\
\hline Conformity coefficient $([\overline{46} \mid)$ & $\mu_{F P}=\frac{2\left(\Re\left(\left\langle S_{H H} S_{V V}^{\star}\right\rangle\right)-\left\langle\left|S_{H V}\right|^{2}\right\rangle\right)}{\left\langle\left|S_{H H}\right|^{2}\right\rangle+2\left\langle\left|S_{H V}\right|^{2}\right\rangle+\left\langle\left|S_{V V}\right|^{2}\right\rangle}$ \\
\hline $\begin{array}{l}\text { Determinant of the sample covariance matrix } \\
\text { ([2] [3|) }\end{array}$ & $\operatorname{det}\left(\boldsymbol{C}_{(F P)}\right)$ \\
\hline $\begin{array}{l}\text { Span of the sample covariance matrix } \\
([45])\end{array}$ & $\operatorname{span}\left(\boldsymbol{C}_{(F P)}\right)$ \\
\hline Copolarization difference $(3,47)$ & $\mathrm{PD}=\left\langle\left|S_{H H}\right|^{2}\right\rangle-\left\langle\left|S_{V V}\right|^{2}\right\rangle$ \\
\hline Cross-polarization ratio & $P_{X}=\frac{\left\langle\left|S_{H V}\right|^{2}\right\rangle}{\left\langle\left|S_{H H}\right|^{2}\right\rangle+\left\langle\left|S_{V V}\right|^{2}\right\rangle}$ \\
\hline Eigenvalues of the sample covariance matrix & $\lambda_{1}>\lambda_{2}>\lambda_{3}$ \\
\hline Entropy ([9] [46] [45] 48] [49] [50] [51]) & $H=-\sum_{i=1}^{3} p_{i} \log _{3} p_{i}, \quad p_{i}=\frac{\lambda_{i}}{\sum_{i=1}^{3} \lambda_{i}}$ \\
\hline Mean $\alpha$ angle of eigenvectors & $\langle\alpha\rangle=\sum_{i=1}^{3} p_{i} \cos ^{-1}\left(\boldsymbol{e}_{i}(1)\right)$ \\
\hline Anisotropy & $A=\frac{\lambda_{2}-\lambda_{3}}{\lambda_{2}+\lambda_{3}}$ \\
\hline Polarization fraction & $P F=1-\frac{\lambda_{3}}{\lambda_{1}+\lambda_{2}+\lambda_{3}}$ \\
\hline Pedestal height & $P H=\frac{\lambda_{3}}{\lambda_{1}}$ \\
\hline
\end{tabular}

with increasing wind speed and to increase with frequency (Bragg wavenumber), oil viscosity, and thickness [41] [42]. The damping ratio is a function of the Bragg coefficients and the 2-D wavenumber spectral density of the ocean surface roughness [1]. The change in the effective dielectric constant decreases the backscatter power only if the oil spill is sufficiently thick or if the oil slick is mixed into the water in high enough concentration in a layer below the surface [13]. The oil slicks in our case are quite small in volume and areal extent. In [19], the authors estimated thickness of the emulsion slicks to be in the range $1.3-1.7 \mu \mathrm{m}$ and $0.7 \mu \mathrm{m}$ for the plant oil in the UAVSAR scene acquired at 06:26 UTC. The expected penetration depth for L-band radar is much higher than these thicknesses. The radiation penetrates to the underlying seawater surface from which it scatters, and the ratio between the Bragg coefficients between the open water and the oil slick is approximately unity because the scattering occurs mainly from the water interface [1]. The damping ratios are located in the first frame in Table IV. Damping ratios extracted from L-band UAVSAR imagery covering the Deepwater Horizon oil spill were discussed in [1]. It was shown that the $\mathrm{HH}$ was dampened slightly less than the $\mathrm{VV}$ and $\mathrm{HV}$.

The copolarization power ratio is the ratio between the intensity of the complex scattering coefficients in the $\mathrm{HH}$ and VV channels. This feature has been found useful in several studies, e.g., [1] [2]. In the tilted Bragg model, the copolarization power ratio is independent of the damping of gravity-capillary waves by the oil and is sensitive to the dielectric constant, the large-scale roughness, and the incidence angle [1].

Table IV: Polarimetric features related to factors in the twoscale Bragg scattering theory discussed in Section III This set-up is based on the discussion of polarimetric features in Section V]. Note the incidence angle is either $\theta$ and/or the local $\theta_{i}$ (see Section II).

\begin{tabular}{|c|c|c|}
\hline Factors & \multicolumn{2}{|c|}{ Polarimetric features } \\
\hline $\begin{array}{l}\text { - Large-scale roughness } \\
\text { - Small-scale roughness } \\
\text { - Incidence angle } \\
\text { - Dielectric properties }\end{array}$ & $\begin{array}{l}\text { FP } \\
-- \\
\text { HP }\end{array}$ & $\begin{array}{l}\zeta_{H H}, \zeta_{V V}, \zeta_{H V}, r_{C O}, \\
i_{C O}, \operatorname{det}\left(\boldsymbol{C}_{(F P)}\right), \operatorname{span}\left(\boldsymbol{C}_{(F P)}\right), \\
\mathrm{PD}, \lambda_{1}, \lambda_{2}, \lambda_{3}-\overline{-}-\bar{\lambda}_{-}-\bar{q}_{1},--- \\
-\zeta_{R H}, \bar{\zeta}_{R V}, \zeta_{R R}, \bar{\zeta}_{R L}, q_{0},-\lambda_{2} \\
q_{2}, q_{3}, \lambda_{1}^{H P}, \lambda_{2}^{H P} \\
\operatorname{det}\left(\boldsymbol{C}_{(R H, R V)}\right), \operatorname{det}\left(\boldsymbol{C}_{(R R, R L)}\right)\end{array}$ \\
\hline $\begin{array}{l}\text { - Large-scale roughness } \\
\text { - Incidence angle } \\
\text { - Dielectric properties }\end{array}$ & $\begin{array}{l}\text { FP } \\
-- \\
\text { HP }\end{array}$ & $\begin{array}{l}\gamma_{C O}, \rho_{C O}, \mu_{F P}, \mathrm{P}_{X}, \phi_{C O}, \\
\mathrm{H},\langle\alpha\rangle, \mathrm{A}, \mathrm{PF}, \mathrm{PH} \\
-\overline{\mathrm{DoP}} \bar{\chi}, \bar{\chi}, \bar{\delta}, \bar{\alpha}, \bar{\mu}_{E}, \bar{\gamma}_{R V / R H},---- \\
\rho_{(R H, R V)}, \phi_{(R R, R L)}, \rho_{(R R, R L)}, \\
\phi_{(R H, R V)}, H_{w}, \mu_{H P}\end{array}$ \\
\hline
\end{tabular}

Based on this, the copolarization power ratio is placed in 
the last frame in Table IV. In general, the ocean wave spectrum is independent of the polarizations. Following this, all the polarimetric features that are composed of ratios are independent of the ocean wave spectrum and depend only on the angles related to the slope and tilt, incidence angle, and the dielectric properties.

The real part of the copolarization cross product has been shown to be a useful feature for detecting oil. Skrunes et al. [2] observed a decrease in correlation when moving from slickfree to slick-covered areas when using spaceborne SAR data. A difference in correlation was also observed using one of the UAVSAR scenes from the NORSE2015 experiment [19]. The physical mechanism behind the change in the correlation of the copolarization channels is yet unknown, but Bragg versus nonBragg scattering, lower backscatter response for slick-covered surface, and change in scattering mechanism are some theories related to this feature. In the tilted Bragg model, no terms cancel out for this feature, hence this feature is in the top-row of Table IV, depending on all the factors in the tilted Bragg model.

Another feature frequently used for slick detection is the standard deviation of the phase difference between the copolarization scattering coefficients. This feature is related to the target's properties and measures the degree of correlation between $\mathrm{S}_{H H}$ and $\mathrm{S}_{V V}$ [52]. The authors in [4] used this feature to characterize the scattering return from oil spills and biogenic slicks. They differentiated the mineral oil from the clean sea under low to moderate wind conditions, and found higher values of the standard deviation of the phase difference for the mineral oil. In [4] [43], the authors observed that low values of this feature represented the presence of Bragg scattering, and that an increase in this feature indicated departure from the Bragg regime. However, there is a lack of research on how this feature behaves when using high SNR SAR data. As discovered in [1] and [19], Bragg scattering was observed for the oil slick regions as well as in the open water areas. Therefore, using this feature to separate the oil from open water could be a challenging task, as similar scattering mechanism might be present in both the regions. The phase difference is located in the lower panel in Table IV, this is because this feature contains a ratio between the imaginary and real part of the copolarization correlation coefficients (see, e.g., [53]), making it independent of the ocean wave spectrum.

The magnitude of the copolarization correlation coefficient $\left(\rho_{(H H, V V)}\right)$ is a multipolarization feature with values between 0 and 1. Low values of $\rho_{(H H, V V)}$ indicate depolarization effects. These effects are sensitive to the presence of a complex surface, multiple scattering surface layers and/or system noise [53]. This feature will be a function of the root mean square (RMS) slope (large-scale roughness), dielectric constant, and the incidence angle [2] (as shown in Table IV). Studies related to this feature have found low values (low $\mathrm{HH}$ VV correlation) for oil covered areas, and high values for open water regions using both $\mathrm{C}$ - and X-band SAR [2] [44].

Another polarimetric feature that uses multipolarization data is the determinant of the sample covariance matrix. This feature is also similar to the geometric intensity (defined in [2]). The authors in [2] discovered that the geometric intensity gives good contrast between oil slicks and sea for both $\mathrm{X}$ - and C-band SAR data with relatively high incidence angles. They also discovered lower values of this feature for slick-covered areas compared to slick-free areas. Neither the span nor the determinant of the sample covariance matrix contain ratios of scattering coefficients, hence these features are given in the top-row in Table IV

Features related to the eigenvalues and the eigenvectors of the FP sample covariance matrix are also considered. The ones evaluated in this study, for the FP case, are the eigenvalues, entropy $(\mathrm{H})$, anisotropy $(\mathrm{A})$, polarization fraction $(\mathrm{PF})$, and the mean alpha angle $(\langle\alpha\rangle)$. The entropy contains information regarding the degree of randomness of the scattering process, while the anisotropy represents the relative importance of the second and third eigenvalue [54]. These features are all composed of ratios of eigenvalues extracted from the covariance matrix, and we can therefore assume, following the two-scale Bragg model, that these features are independent on the smallscale roughness, and is only a function of the large-scale roughness, the dielectric properties, and incidence angle (as reflected in the second frame in Table IV .

\section{B. Hybrid-polarity features}

The polarimetric features extracted from the simulated HP data used in this analysis are given in Table $\mathrm{V}$. with the corresponding references given in parentheses. The Stokes vector is a popular feature when analyzing HP data. The expression for the Stokes vector for linear receive polarization is given in Table $\mathrm{V}$. Each of the Stokes parameters are tested in this study, where the first Stokes parameter $\left(q_{0}\right)$ is the total power, the second, $q_{1}$, is the power in the linear horizontal or vertical polarization, $q_{2}$ is the power in the linearly polarized components at tilt angles $45^{\circ}$ and $135^{\circ}$, and $q_{3}$ is equal to the power in the left-handed and right-handed circular polarizations [55]. The authors in [18] showed that the imaginary part of the $\mathrm{RH}$ and $\mathrm{RV}\left(q_{3}\right)$ follows the same trend as the copolarization cross product, which is lower correlation for the oil slicks than for clean water. Following the two-scale Bragg model of the HP data, the $q_{0}$ and $q_{3}$ are a function of the tilt angles, wave spectrum, incidence angle, and the dielectric properties. While $q_{1}$ and $q_{2}$ depends on the same terms, in addition to the rotation angle (as see in Section [II-B].

Child parameters of the Stokes vector evaluated in this study are the degree of polarization (DoP), the ellipticity angle $(\chi)$, the circular-polarization ratio $\left(\mu_{E}\right)$, the relative phase $(\delta)$, and the alpha angle $(\alpha)$. The DoP has been extensively used in the literature (see, e.g., [10] [12] [57]), and describes the degree of depolarization in the measured signal backscattered from a given surface element. The DoP has also been classified as a good parameter for oil spill detection in [57]. The $\chi$ feature is used in the $m-\chi$ (where $m$ is DoP) decomposition [59], and this feature could help in distinguishing the even versus odd bounce scattering. If the open water and the oil slicks posses' different scattering mechanisms the resulting separability would be high for this feature. The features $\chi$, $\mu_{E}, \delta, \alpha$ are ratios of the Stokes parameters. In the two-scale Bragg model (see Section III) these features are independent of 
Table V: Overview of the investigated HP (with right circular transmits and linear receive) features (computed using a mask of $15 \times 60$ pixels). The references included are examples of studies where the features have been investigated for oil spill observation. The "circular-linear" basis denotes the HP features, while the "circular-circular" denotes the HP features projected into the circular transmit and circular receive basis.

\begin{tabular}{|c|c|}
\hline \multicolumn{2}{|c|}{ HP Features (based on measuring RH and RV) } \\
\hline Name & Formula \\
\hline Stokes vector $(|6||56|)$ & $\boldsymbol{q}=\left[\begin{array}{c}q_{0} \\
q_{1} \\
q_{2} \\
q_{3}\end{array}\right]=\left[\begin{array}{c}\left\langle\left|S_{R H}\right|^{2}+\left|S_{R V}\right|^{2}\right\rangle \\
\left\langle\left|S_{R H}\right|^{2}-\left|S_{R V}\right|^{2}\right\rangle \\
2 \Re\left\langle\left(S_{R H} S_{R V}^{\star}\right)\right\rangle \\
-2 \Im\left\langle\left(S_{R H} S_{R V}^{\star}\right)\right\rangle \\
\text { (circular-linear) }\end{array}\right]=\left[\begin{array}{c}\left\langle\left|S_{R R}\right|^{2}+\left|S_{R L}\right|^{2}\right\rangle \\
2 \Re\left\langle\left(S_{R R} S_{R L}^{\star}\right)\right\rangle \\
2 \Im\left\langle\left(S_{R R} S_{R L}^{\star}\right)\right\rangle \\
-\left\langle\left|S_{R L}\right|^{2}-\left|S_{R R}\right|^{2}\right\rangle\end{array}\right]$ \\
\hline Degree of polarization $(\mid 10\rceil \mid 12\rceil|57|)$ & $\mathrm{DoP}=\frac{\sqrt{q_{1}^{2}+q_{2}^{2}+q_{3}^{2}}}{q_{0}}$ \\
\hline Ellipticity angle $(10,57 \mid)$ & $\chi=\frac{1}{2} \sin ^{-1}\left(-\frac{q_{3}}{\operatorname{DoP} q_{0}}\right)$ \\
\hline Relative phase ( $|\overline{7}| \overline{\mid})$ and alpha angle $(|\overline{58}|)$ & $\delta=\frac{1}{2} \tan ^{-1}\left(\frac{q_{3}}{q_{2}}\right) \quad \alpha=\frac{1}{2} \tan ^{-1}\left(\frac{q_{1}+q_{2}}{q_{3}}\right)$ \\
\hline Damping ratio & $\begin{array}{c}\zeta_{i j}=10 \log _{10}\left(\frac{\left\langle I_{i j}^{(\text {sea })}\right\rangle}{\left\langle I_{i j}^{\text {slick })}\right\rangle}\right), \text { where } I_{i j}=\left|S_{i j}\right|^{2} \\
(i, j) \in((\mathrm{R}, \mathrm{H}) \vee(\mathrm{R}, \mathrm{V})) \vee((\mathrm{R}, \mathrm{R}) \vee(\mathrm{R}, \mathrm{L})) \\
\quad(\text { circular-linear }) \quad(\text { circular-circular })\end{array}$ \\
\hline Circular-polarization ratio $([9])$ & $\mu_{E}=\frac{q_{0}-q_{3}}{q_{0}+q_{3}}\left(\right.$ also equal to: $\left.\gamma_{R R / R L}=\frac{\left\langle I_{R R}\right\rangle}{\left\langle I_{R L}\right\rangle}\right)$ \\
\hline Hybrid-polarization power ratio $(35 \mid)$ & $\gamma_{R V / R H}=\frac{\left\langle I_{R V}\right\rangle}{\left\langle I_{R H}\right\rangle}$ \\
\hline Correlation coefficient $([\overline{10}][\overline{9}])$ & $\begin{array}{cc}\rho_{(R H, R V)}=\frac{\left|\left(S_{R H} S_{R V}^{\star}\right)\right|}{\sqrt{\left\langle\left|S_{R H}\right|^{2}\right\rangle\left\langle\left|S_{R V}\right|^{2}\right\rangle}} \quad \text { (circular-linear) } \\
\rho_{(R R, R L)}=\frac{\left|\left(S_{R R} S_{R L}^{\star}\right)\right|}{\sqrt{\left\langle\left|S_{R R}\right|^{2}\right\rangle\left\langle\left|S_{R L}\right|^{2}\right\rangle}} \quad \text { (circular-circular) }\end{array}$ \\
\hline Standard deviation of the phase difference & $\begin{array}{l}\phi_{(R H, R V)}=\sqrt{\left\langle\left(\phi_{R H}-\phi_{R V}\right)^{2}\right\rangle+\left(\left\langle\phi_{R H}-\phi_{R V}\right\rangle\right)^{2}} \quad \text { (circular-linear) } \\
\phi_{(R R, R L)}=\sqrt{\left\langle\left(\phi_{R R}-\phi_{R L}\right)^{2}\right\rangle+\left(\left\langle\phi_{R R}-\phi_{R L}\right\rangle\right)^{2}} \quad \text { (circular-circular) }\end{array}$ \\
\hline Eigenvalues & $\lambda_{1,2}^{H P}=q_{0} \pm \sqrt{q_{1}^{2}+q_{2}^{2}+q_{3}^{2}}$ \\
\hline Entropy (wave entropy) $([\overline{9} \mid)$ & $H_{w}=-\sum_{i=1}^{2} p_{i} \log _{2} p_{i}, \quad p_{i}=\frac{\lambda_{i}^{H P}}{\sum_{i=1}^{2} \lambda_{i}^{H P}}$ \\
\hline Conformity coefficient $([\overline{10}][\overline{46}])$ & $\mu_{H P}=\frac{2 \Im\left\langle S_{R H} S_{R V}^{\star}\right\rangle}{\left\langle\left|S_{R H}\right|^{2}\right\rangle+\left\langle\left|S_{R V}\right|^{2}\right\rangle}$ \\
\hline Determinant of the covariance matrix & $\begin{array}{l}\operatorname{det}\left(\boldsymbol{C}_{(R H, R V)}\right)=\operatorname{det}\left(\left[\begin{array}{cc}\left\langle\left|S_{R H}\right|^{2}\right\rangle & \left\langle S_{R H} S_{R V}^{\star}\right\rangle \\
\left\langle S_{R V} S_{R H}^{\star}\right\rangle & \left\langle\left|S_{R V}\right|^{2}\right\rangle\end{array}\right]\right) \text { (circular-linear) } \\
\operatorname{det}\left(\boldsymbol{C}_{(R R, R L)}\right)=\operatorname{det}\left(\left[\begin{array}{cc}\left\langle\left|S_{R R}\right|^{2}\right\rangle & \left\langle S_{R R} S_{R L}^{\star}\right\rangle \\
\left\langle S_{R L} S_{R R}^{\star}\right\rangle & \left\langle\left|S_{R L}\right|^{2}\right\rangle\end{array}\right]\right) \quad \text { (circular-circular) }\end{array}$ \\
\hline
\end{tabular}

the ocean wave spectrum (the damping of the gravity-capillary waves by oil). This indicates that these features are function of the dielectric constant, the incidence angle, and the large-scale roughness (see second frame in Table IV.

The hybrid-polarization power ratio is the ratio between the intensity of the simulated complex scattering coefficients in the RH and RV channels. Since the copolarization intensities have higher response than the cross-polarization intensity, the hybrid-polarization ratio is expected to have approximately the same behavior as the copolarization ratio discussed in the previous section. Hence, this feature is also independent of the ocean wave spectrum. The standard deviation of the phase difference between the RH and RV scattering coefficients has been found to be good feature for oil spill detection [10]. We also test the standard deviation of the phase difference between the RR and RL scattering coefficients.

The magnitude of the hybrid-polarization correlation coefficients are also considered, both in circular-linear and circularcircular basis, i.e., $\rho_{(R H, R V)}$ and $\rho_{(R R, R L)} \cdot \rho_{(R R, R L)}$ was introduced in [10], and the authors named it the HP coherence measure. The authors in the same article demonstrated this feature on five Radarsat- 2 scenes covering various types of oil. From the figures in [10] one can see that low values of $\rho_{(R R, R L)}$ are present for the oil slick regions and high values for the open water areas, which is the same behavior as the $\rho_{(H H, V V)}$. The authors concluded that this feature could suppress some lookalikes caused by low wind and also generated good slick-sea contrast. In [9], the authors also found low values of $\rho_{(R H, R V)}$ for oil covered areas and high values for open water using both L- and C-band SAR. These features are located in the last row of Table [V], where $\rho_{(R H, R V)}$ and $\rho_{(R R, R L)}$ are independent of the small-scale roughness since these features are composed of ratios.

The conformity coefficient is a multipolarization feature containing both cross- and copolarization intensities and correlation. The FP variant of this feature can be seen in Table III. and to calculate this feature the reflection symmetry assumption must be made. In [46], the authors stated that this 
feature can be used to distinguish different scattering mechanisms of ambient sea surfaces and slicks. They concluded that when $\mu$ was positive, Bragg scattering took place, and the pixels producing such values was classified as slick free area. Negative values was defined as non-Bragg scattering and thus classified as slick-covered areas. However, the authors of [1] discovered that Bragg scattering was present within the slickcovered areas, and this feature might therefore not follow the theory suggested by [46] for separating the oil slicks from open water using the UAVSAR data. The conformity coefficient, $\rho_{(R H, R V)}$, and $\rho_{(R R, R L)}$ contain ratios of scattering coefficients, and since the ocean wave spectrum is polarization independent, these features become independent of the ocean wave spectrum, as given in the second frame in Table IV

The determinant of the simulated sample HP covariance matrix, both in the circular-linear and circular-circular basis, is also evaluated. The authors did not find studies related to these two features in the HP scenario. Unfortunately, the HP sample covariance matrix is two dimensional, and the anisotropy is not available since it requires the two minimum eigenvalues from a three dimensional matrix. This is only possible if a reconstruction of a pseudo FP covariance matrix is performed. The HP entropy (known as the wave entropy), however, can be calculated from the Stokes vector. This was done for an oil spill study in [9], and was found to have the same behavior as the FP entropy, that is, large for slick-covered areas and low for slick-free areas.

\section{RESULTS AND DisCUSSIONS}

In the following subsections, the results obtained from analysis of the UAVSAR time series are presented. The simulated HP features are compared to the FP features in terms of slick detectability. The change in separability based upon the best FP and HP features as a slick evolves naturally on the sea surface is also discussed. The results obtained from the simulated HP features are also compared to previous findings.

\section{A. Noise analysis}

As is already known, the returns from the oil slicks are low, and hence a noise analysis of the data used is important. The minimum backscattered signal that can be detected from a given surface element is dependent on the system's noise floor. The noise floor, related to the noise equivalent sigma zero (NESZ), is extremely low for the UAVSAR instrument (NESZ in the range -48 to $-33 \mathrm{~dB}[5]$ ) compared to other sensors like Radarsat-2 (NESZ in the range -27.5 to -43 $\mathrm{dB}[60]$ ), and TerraSAR-X (NESZ in the range -19 to $26 \mathrm{~dB}$ [61]). Several studies of the effect of the NESZ on radar-dark surfaces like oil slicks have been conducted using spaceborne SAR sensors [2] [3] [62], and have shown that a large part of the cross-polarization return and also some part of the copolarization return from oil slicks are near or even below the instrument noise floor. RISAT-1 is the first spaceborne satellite that offers the circular or HP imaging mode. Unfortunately, the NESZ is high, $-17 \mathrm{~dB}$, for the RISAT1 FRS-1 mode [63]. A consequence of returns below the NESZ is loss of information, and even though the slick can

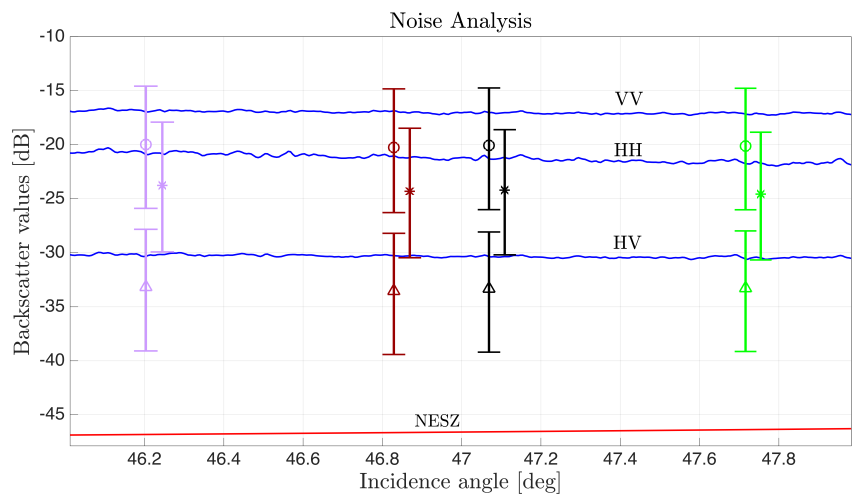

Figure 6: Noise analysis from the UAVSAR scene taken at 06:26 UTC (ascending). The 5th, 50th and 95th percentiles are calculated for each slick region, and plotted with a vertical line from the 5th to the 95th percentile, and a symbol indicating the 50th percentile: a circle for $\mathrm{VV}$; a star for $\mathrm{HH}$; and a triangle for HV. The lines for $\mathrm{HH}$ are slightly shifted to higher incidence angle to improve the discrimination in the plot. The blue continuous lines show the 50th percentile for clean sea samples selected along the range direction.

be detected through comparison to clean water signals above the NESZ, oil spill characterization may not be possible.

The NESZ for the UAVSAR varies between $-48 \mathrm{~dB}$ at the point of maximal antenna gain and $-33 \mathrm{~dB}$ in the far range [5]. Such a low NESZ is important for our application as our goal is analyzing the backscattered response from the various slicks, in addition to simulating the HP data from the FP data, resulting in a mix of co- and cross-polarization channels and a $3 \mathrm{~dB}$ power loss when simulating the HP scattering vector [8]. The HP intensities are still above the noise floor for all the UAVSAR scenes used in this analysis. We demonstrate this using one scene in Fig. 6, and the other scenes show a similar trend.

Fig. 6 shows an example of the noise analysis we performed. The 5th, 50th, and 95th percentiles of the HH, VV, and HV intensities are calculated for each region. No multilooking and incidence angle correction has been done prior to the noise analysis in order to show the characteristics of the different intensities of the actual measured values at the highest instrument resolution. The 50th percentiles are indicated by various symbols depending on the polarization used. The blue continuous lines show the 50th percentile for clean sea samples selected along the range direction for the three intensities. Following [1], an acceptable return was suggested to be $6 \mathrm{~dB}$ above the noise floor, i.e., $20 \%$ of the measured signal is noise, and $80 \%$ is the signal backscattered. The NESZ is indicated by the red continuous line in Fig. 6. The NESZ as a function of incidence angle is found in [5]. The $\mathrm{HH}$ intensities are slightly below the VV intensities, but well above the NESZ $+6 \mathrm{~dB}$ limit. This is also the case for the other UAVSAR scenes used in this analysis. Hence, the noise should not have a significant impact on the various polarimetric features extracted from the UAVSAR scenes. Similar results are found in [19] for one UAVSAR acquisition from the NORSE2015 experiment. 


\section{B. Slick separability based on FP and HP features}

The polarimetric feature values are calculated for each region (different slicks and open water), and their statistical properties used as input in calculation of the JM separability measure. Fig. 7 to 10 show charts of the mean JM separability for all of the polarimetric features for slick versus open water. The separability between the various slicks is not shown because the JM separability is below 0.6 for all cases. Note that the JM separability is calculated between each slick and its corresponding subset of open water regions (as seen in Fig. 57. This result in several JM distances, and the average of these are shown in the color charts in Fig. 7 to 10 . Due to space limitation, the standard deviation of the JM measures are left out, but these are shown later for the features with the maximum JM separability. Note that the features in the color charts are sorted so that the JM separability decreases from the top of the chart to the bottom. In Fig. 7 to 10 the red color indicates the highest separability between the slick and the open water. Red, orange, yellow, green, dark, and light blue colors indicate separability, in decreasing order. Using color charts, we obtain a good overview of all the polarimetric features, and can more easily identify the best ones.

The FP color chart in Fig. 7, representing the average separability between PO and the OW regions, is the one that contains highest separability for the various features along the time series compared to the other emulsion slicks and open water (both for FP and HP). The FP features that provide high separability between the plant oil and open water are the $\zeta_{X}$ (damping ratio) with 7 red and 10 orange cells along the time series, and second is the $\lambda_{3}$ (minimum eigenvalue) with 9 red and 7 orange cells. In other words, using the $\zeta_{X}$ feature the plant oil can be differentiated from open water in 17 out of 18 scenes with relatively high separability.

These two features are also among the best at providing high separability for the emulsion slicks. Considering the FP separability color charts for E40, both the $\zeta_{X}$ and $\lambda_{3}$ feature give high separability for three scenes in the times series. For the two first scenes, several FP features can be used to distinguish either E40 or E60 from the open water regions. The E40 has high separability $(\mathrm{JM}>0.8)$ in 8 out 18 scenes using various features, while the E60 has high separability in 9 out of 18 scenes. The $\zeta_{V V}$ and the PF features provide higher detectability of E60 than E40. The FP color chart representing the separability between E80 and OW is given in Fig. 10. Here, the first acquisition at 05:32 UTC is not included because this oil had not been released. This color chart contains several orange cells, more than the E40 and E60 FP color charts, which indicates higher overall detectability of E80 than E60 and E40. Again, the $\zeta_{X}$ and $\lambda_{3}$ stands out, followed by $\operatorname{det}\left(\boldsymbol{C}_{(F P)}\right)$, PF, and PD. A more in-depth analysis of how the JM changes with time for the various oil slicks is given in the next section. The FP features that are not able to separate the various slicks from open water are $\gamma_{C O}$, $P_{X}, \phi_{C O}, \rho_{C P}, \mathrm{PH}, \mu_{F P}, \mathrm{H},\langle\alpha\rangle$, and $\mathrm{A}$, according to the threshold that is set for the JM distance. One previous study related to the use of UAVSAR L-band for oil spill observation (Deepwater Horizon oil spill) was presented in [1]. The authors in [1] discovered that the $\langle\alpha\rangle$ was sensitive to the change in the dielectric constant rather than damping of the ocean waves. To detect the oil using $\langle\alpha\rangle$ it is required that the oil must mix with the ocean to create an intermediate dielectric layer and/or the oil slick is sufficient thick enough (see section If. The low separability values of $\langle\alpha\rangle$ in our case might indicate that such a layer was non-present. The authors in ([1]) also discovered, based on the entropy $(\mathrm{H})$, that both the oil slicks and open water had one dominant scattering mechanism, namely the Bragg scattering. Therefore, it is challenging to use the entropy to separate the oil slicks from the clean sea, as the same scattering characteristics might be present. Although it has been suggested that the entropy is sensitive to slickcovered surfaces (high entropy for slick-covered surface and low entropy for slick-free surface) in several studies using spaceborne SAR data under various wind conditions [32] [45] [48] [51], this is not the case for our dataset. The set of features that are incapable of separating the four oil slicks from the open water region are all located in the lower panel in Table [V] The top best features for detecting the various oil slicks are located in the top panel in Table IV This indicates that the features independent on the small-scale roughness show poor detection capabilities, while features containing the small-scale roughness shows good detectability.

Previous studies have found that $\operatorname{det}\left(\boldsymbol{C}_{(F P)}\right)$ (only using copolarization products) and $r_{C O}$ are best at distinguishing biogenic slicks from mineral oil under low wind conditions, in this case using Radarsat-2 C-band data [2]. The $\operatorname{det}\left(\boldsymbol{C}_{(F P)}\right)$ and $r_{C O}$ in our case have JM above 0.5 in all scenes, but they do not separate as well as the $\zeta_{X}$ and $\lambda_{3}$ features. The same study ([2]) did exclude features that contained the cross-polarization scattering coefficients because they had a large part of the signal below the noise floor. Using the UAVSAR data, the noise is no longer an issue for the crosspolarization scattering components, and we are now able to see the usefulness of the cross-polarization feature, for example the high separability of the $\zeta_{X}$ and $\lambda_{3}$ features. The reasons why the cross-polarization feature is the best for detecting the oil should be further investigated. One theory could be that the cross-polarization intensity is closer to the noise floor compared to the copolarization intensities. Other theories could be related to the depolarization effects caused by the dielectric properties within the oil or that the tilt angles are larger for high wind conditions. The good potential of the cross-polarization feature was also highlighted using UAVSAR data from the Deepwater Horizon oil spill [1], and also in one of the UAVSAR scenes from the NORSE2015 experiment [64].

The right panels in Fig. 7 - 10 show the color charts for the HP features along the time series. Fewer red colored cells are observed for the PO vs. OW HP color chart compared to the PO vs. OW FP color chart. Unfortunately, the polarimetric features containing the cross-polarization component are no longer possible to separate out in the HP mode. The HP features that have high separability between PO and OW, in decreasing order, are the $\zeta_{R R}, \zeta_{R V}, q_{1}, q_{0}, \lambda_{1}, \zeta_{R L}$, $\operatorname{det}\left(\boldsymbol{C}_{(R H, R V)}\right), \operatorname{det}\left(\boldsymbol{C}_{(R R, R L)}\right)$, and $q_{3}$, respectively. The same features also provide high separability for the emulsion 
slicks. The HP features that are comparable to the FP features show similar colors of the JM separability, for example the $\zeta_{R V}$ and $\zeta_{R H}$ show similar separability values as the $\zeta_{V V}$ and $\zeta_{H H}$ for all the various slicks. The $\gamma_{R V / R H}, \phi_{(R H, R V)}$, $\phi_{(R R, R L)}, \rho_{(R R, R L)}, \rho_{(R H, R V)}, \mu_{H P}, \mathrm{H}_{w}, \mathrm{DoP}, \chi$, and $\mu_{E}$ are features that achieve low separability between the various slicks and open water for all the UAVSAR scenes. This corresponds well with the set-up in Table IV, where the features resulting in high separability are dependent on, amongst other, the small-scale roughness, and the features showing low separability are independent of this factor. This was also found for the corresponding FP features, namely $\gamma_{C O}, \phi_{C O}, \mu_{F P}$, and $\mathrm{H}$. Hence, we are left with $12 \mathrm{HP}$ features that perform reasonably well at separating the various oil types from the open water regions. For the emulsions slicks, the E40 vs. OW HP color chart has the minimum number of orange colored cells, followed by E60 vs. OW and E80 vs. OW HP color charts. This is the same behavior as the FP color charts for the emulsion slicks.

Previous studies related to the HP features simulated from spaceborne FP SAR have obtained different results. The authors in [10] used the same dataset as [2], and discovered that $\rho_{(R R, R L)}$ (the authors in [10] named this feature Coh), the conformity index $\mu_{H P}$, and the DoP can be used to detect various types of oil (plant, emulsion, and crude oil), using C-band data under low to moderate wind condition. In [9], the authors concluded based on L- and C-band spaceborne SAR data that the wave entropy $\left(\mathrm{H}_{w}\right)$, circular-polarization ratio $\left(\mu_{E}\right), \rho_{(R R, R L)}$, and the relative intensity of the polarized component to the intensity of the total field could discriminate slick-free, weak-damping slick covered, and strong-damping slick-covered sea surfaces. Additionally, [65] also concluded that the DoP could be used to detect the oil spills from the ocean surface using both C-band SAR and L-band UAVSAR data. The separability observed in our color charts do not agree with the findings in [9] [10] [65], which may be due to several factors, including the high wind, the small slicks, and the fact that the data used in this study are well above the noise floor.

The highest JM separabilities obtained from both the FP and HP features across the entire UAVSAR time series are shown in Fig. 11. The green, pink, red, and black colors represent the highest mean JM separability for the PO vs. OW, E40 vs. OW, E60 vs. OW, and E80 vs. OW, respectively. The FP and HP feature that provides the highest mean JM and the corresponding standard deviation of the JM between the slick and the open water regions (see Fig. 5) are given in Table VI Note that the feature for which the JM separability is highest can change as the slick evolves. For comparison, the highest JM separability obtained using the HP features is plotted as a dashed line in the same figure as the highest JM separability using the FP features. The $\mathrm{x}$-axis represents the time since release of each slick, not the time since the first image was acquired. Because the slicks were not all released simultaneously, the x-axis is shifted for each of the slicks. The UAVSAR time series was collected in two flights, hence each panel in Fig. 11 is divided into two subplots. Additionally, the difference between the ascending and the descending scenes are marked with green and grey colored dots.
There are two ways to evaluate the information in Table VI and Fig. 11 . The first is to study how the separability between the various slicks and the open water regions varies with time and how the weathering process of the emulsion and plant oil slicks affects the detectability. The second way is to identify the polarimetric features that give the highest JM separability as a function of time for the various slicks. Each of these two evaluations will be discussed in the following subsections.

\section{Separability as a function of time}

The first flight covers approximately the first 4 hours after release, while the second flight covers approximately 6.5 8.5 hours following release of the oils, with some variation because the PO was released first and the E80 last. From Fig. 11. we find that the JM separability between the plant oil and the open water regions starts off at 1.2 and then decreases over the next two hours. The separability increases again during the next half hour. During the remaining hours of flight 1 , the separability fluctuates, and in the last hour of flight 2 the separability increases again. From the intensity images in Fig. 1. the PO reaches an equilibrium in terms of shape and size in the beginning of flight 2 , and remain visible throughout the time series.

The separability of the emulsion oils from the open water regions generally decreases with time with some fluctuations along the way. The separability is higher between the E80 and the open water regions along the time series compared to the other emulsion slicks. This might be a result of the higher oil fraction in E80 compared to E60 and E40. The viscosities of the emulsion slicks are characterized by higher viscosity than the natural film [66]. Hence, the emulsion slicks should have a stronger damping of the ocean waves and thus be more detectable than the plant oil. Both Fig. 7 and 11 show that this is not the case, and the plant oil is visible longer on the sea surface than the emulsion slicks. Hence, the plant oil compound used, Radiogreen EBO, might therefore not be a good indicator for simulating biogenic slicks, as already stated in [20].

It is challenging to segment out the emulsion slicks for the UAVSAR scenes in flight 2 , which results in higher presence of the open water in these segments. This might be because of the emulsion slicks have a higher area and higher westerly extent than the plant oil (see Fig. 1). Immediately after release, the emulsion slicks might undergo emulsification, i.e., take in additional water molecules into the oil-water mix [67]. Hence, more water can be mixed with the E80, and higher volume over time. Parts of the oil spill might also break up into drops of varying sizes that are mixed down into the water column (dispersion), and the oil droplets might also resurface. A parallel study investigated the oil slicks' drift using two different oil drift models (see [20]). The model results indicated that the PO entrained more quickly and deeper into the water column compared to the emulsion slicks. Additionally, the PO droplets resurfaced to maintain the observable slick. As a result of the entrainment into the water column, the plant oil was shielded from the wind drag and Stokes drift, which resulted in longer visibility on the 


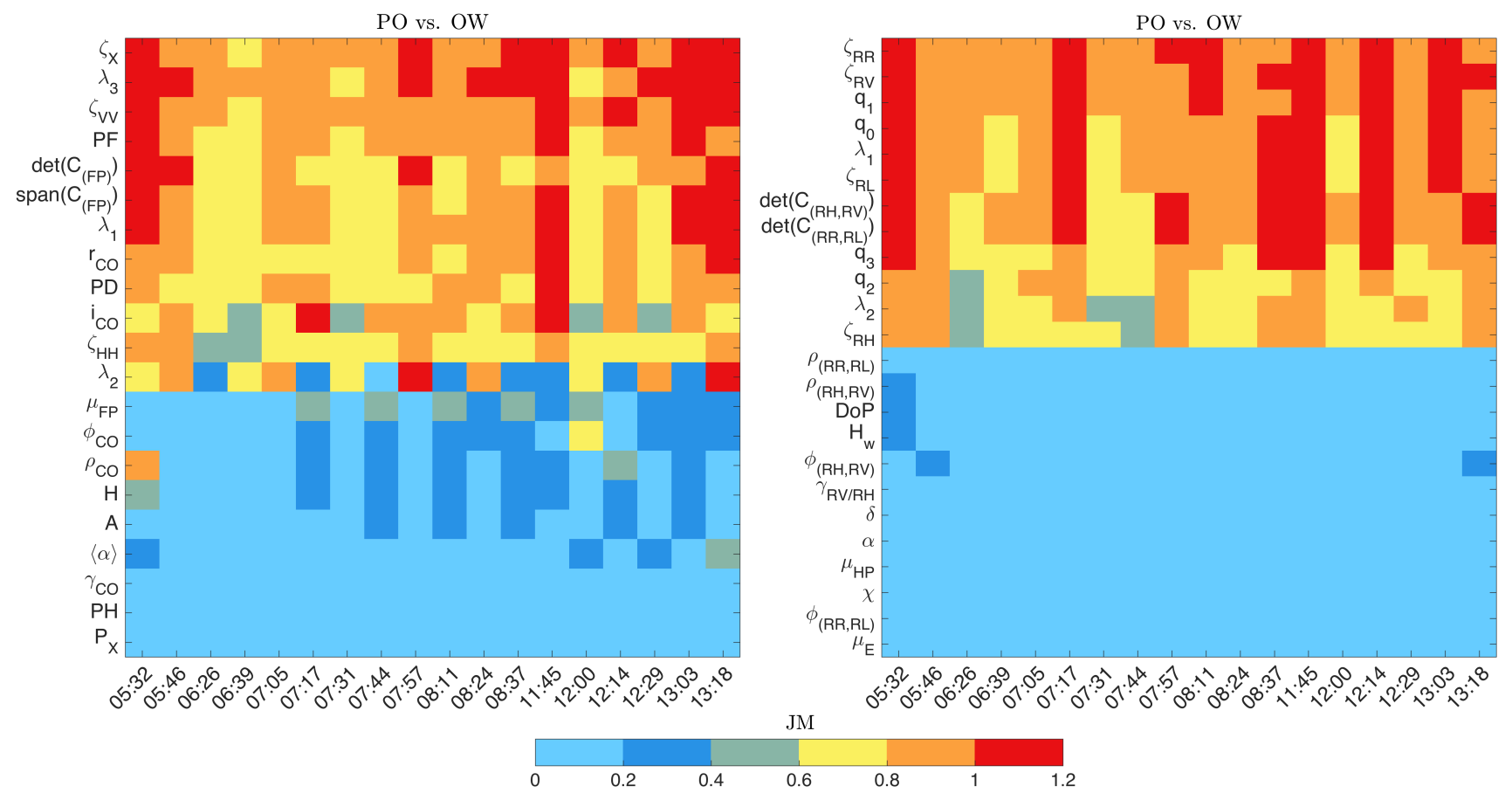

Figure 7: Color charts of the JM separability between PO and OW for the FP (left chart) and the HP (right chart) features. Red, orange, yellow, green, dark, and light blue indicate separability, in decreasing order. The $\mathrm{x}$-axis represents the acquisition time (in UTC).
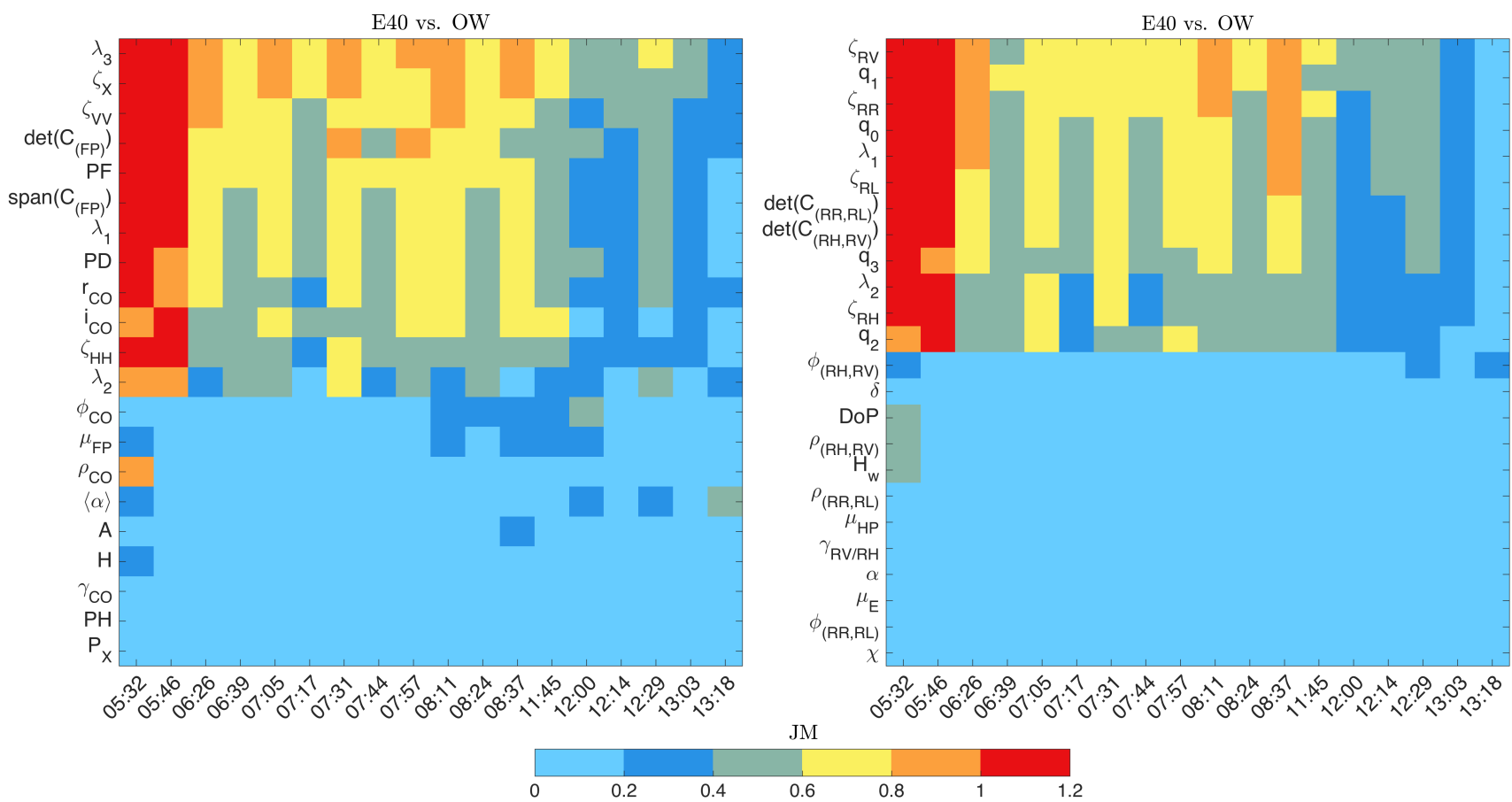

Figure 8: Color charts of the JM separability between E40 and OW for the FP (left chart) and the HP (right chart) features. Red, orange, yellow, green, dark, and light blue indicate separability, in decreasing order. The $\mathrm{x}$-axis represents the acquisition time (in UTC). 


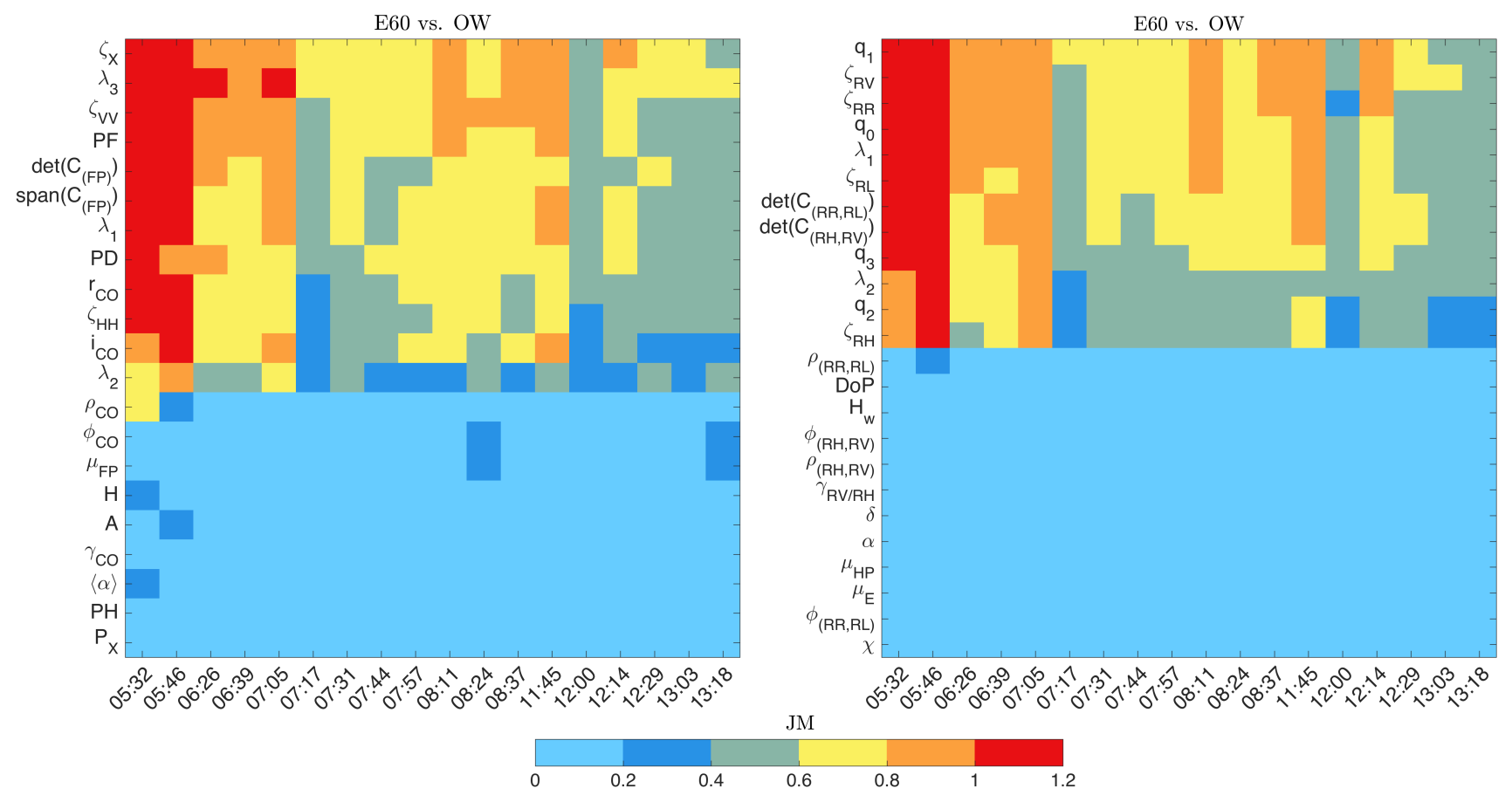

Figure 9: Color charts of the JM separability between E60 and OW for the FP (left chart) and the HP (right chart) features. Red, orange, yellow, green, dark, and light blue indicate separability, in decreasing order. The $\mathrm{x}$-axis represents the acquisition time (in UTC).
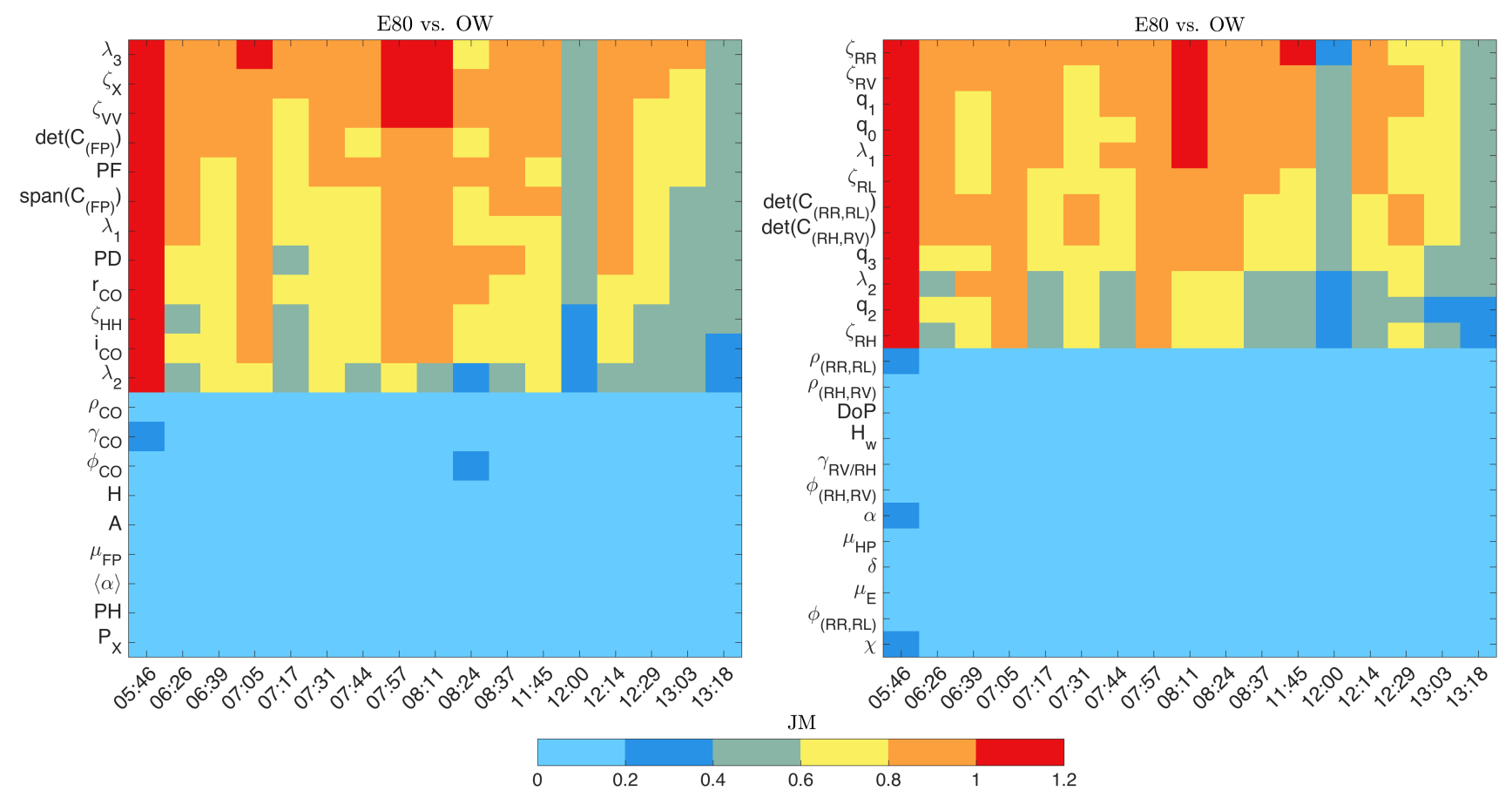

Figure 10: Color charts of the JM separability between E80 and OW for the FP (left chart) and the HP (right chart) features. Red, orange, yellow, green, dark, and light blue indicate separability, in decreasing order. The $\mathrm{x}$-axis represents the acquisition time (in UTC). 
sea surface compared to the emulsion slicks. The same study also compared the E80 slick with the model simulations, and the model results showed that the simulated E80 had 50\% or more of the oil on the surface throughout the simulation, and relatively little penetrated deeper than 10 meters. Hence, the emulsion slicks are more exposed to wind drag and Stokes drift, which results in more spreading than the PO experienced.

The separability as a function of time can be affected by several factors, which include the imaging geometry (difference between ascending and descending), changing wind and ocean state over the time series, weathering processes, and the accuracy of the segmentation for all the scenes. The incidence angle of the plant oil within the scene varies across the times series (see Fig. 2). The incidence angle has a higher variation in flight 2 scenes compared to the flight 1 scenes, which might be the reason for the fluctuations in the separability between the descending and ascending scenes in flight 2. In Appendix A the incidence angle correction applied to the complex scattering vector in the preprocessing of the UAVSAR scenes was discussed. The fact that the incidence angle affects the separability of the PO might be a result of the texture variation with incidence angle (which is not corrected for), and difference in the imaging geometry between ascending and descending. Finding the best incidence angle correction method that can allow comparison across several scenes (especially the ascending and descending in our case) with slightly different incidence angle is a study in itself and should be further investigated.

The plant oil is released to simulate biogenic slicks, and the biogenic slick forms a monomolecular layer [68]. Previous studies have found that biogenic surface films disappears in high wind condition (typically above 7 to $10 \mathrm{~m} / \mathrm{s}$ ) due to entrainment into the underlying water by the breaking waves [16] [68]. The reader is referred to [20] for additional information on how the various oil slicks were transported. In addition, a study on how the polarimetric features are affected by the imaging geometry is on-going.

\section{Polarimetric features with highest separability}

The highest JM separability between the slicks and the open water regions (see Table VI is provided by $\lambda_{3}, \zeta_{V V}$, $\operatorname{det}\left(\boldsymbol{C}_{(F P)}\right)$, PD, $\operatorname{span}\left(\boldsymbol{C}_{(F P)}\right)$, and $\zeta_{X}$ in FP. $\lambda_{3}$ is the feature that provides the highest JM separability most frequently. The majority of the mean JM is around 0.9 to 1.1 , while the standard deviation is around 0.1, which indicates that the JM has a small variation within the open water subsets that are used. The best polarimetric FP features, i.e., $\lambda_{3}, \zeta_{V V}, \operatorname{det}\left(\boldsymbol{C}_{(F P)}\right)$, and $\zeta_{X}$, were also evaluated as a function of time. All showed a similar trend with time as in Fig. 11, but are left out due to space limitation. The best HP features to detect the various oil slicks are $\zeta_{R R}, \zeta_{R V}, \operatorname{det}\left(\boldsymbol{C}_{(R H, R V)}\right), \operatorname{det}\left(\boldsymbol{C}_{(R R, R L)}\right), q_{0}$, and $q_{1}$, and they all have similar separability trend as a function of time to the best FP features. $\zeta_{R V}$ and $\zeta_{R R}$ are the HP features that provide the highest JM separability along the time series.

Overall, the best FP features are $0.6 \%$ better for detecting the E80 compared to the simulated HP features. For the E60 and $\mathrm{E} 40$, the best FP features are $1.6 \%$ and $3.3 \%$ better than

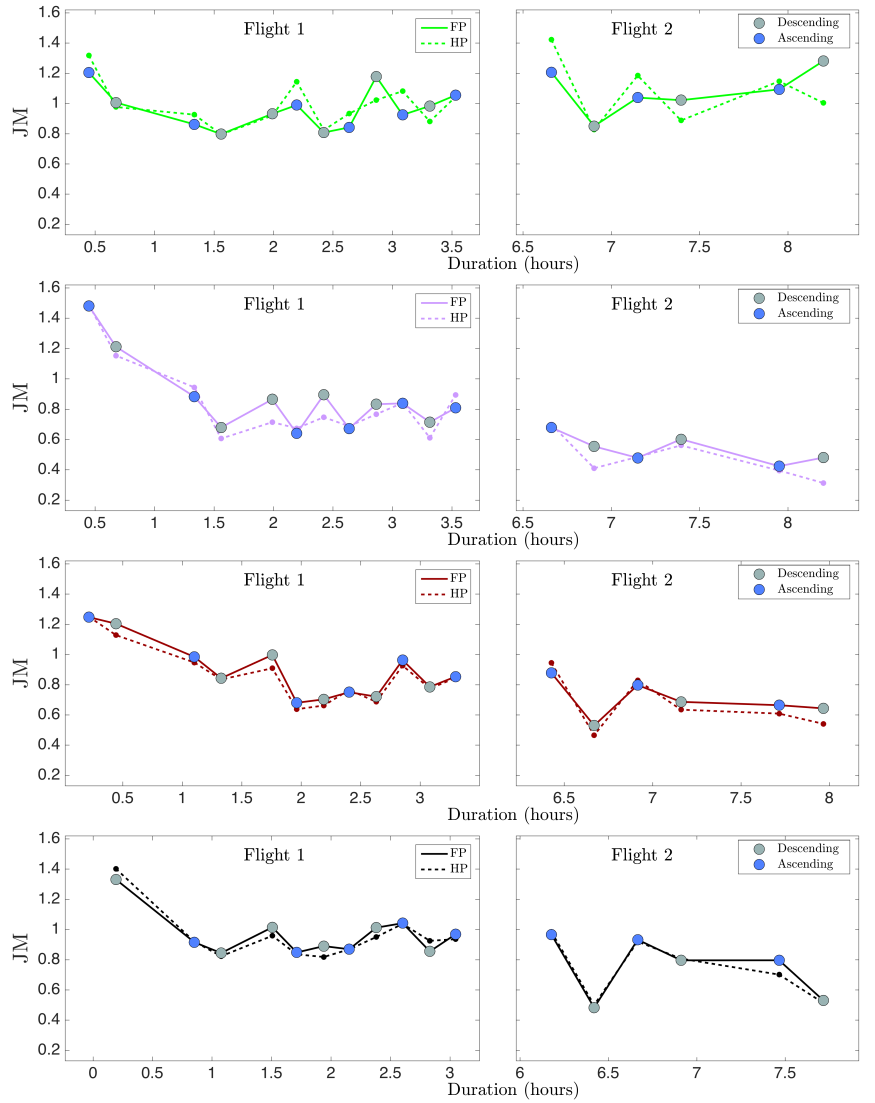

Figure 11: The maximum JM distance obtained from all the polarimetric features along the time series. The points represent the maximum mean JM distance, and the solid (dashed) lines represent the values between these for the FP (HP) features. The green, pink, red, and black color represent the JM separability between the PO vs. OW, E40 vs. OW, E60 vs. OW, and E80 vs. OW. The blue and grey markers represent the ascending and descending scenes.

the HP features for detection. However, for detection of the PO, the best HP features showed $0.8 \%$ better detection ability compared to the FP.

\section{CONCLUSION}

A comparison between FP and simulated HP data from a UAVSAR time series of recently released and evolving oil slicks has been presented. The relative performance of FP and simulated HP in slick detection capability using a wide range of polarization-dependent features has been carefully evaluated using the JM separability.

Overall, the FP features were estimated to be $0-3.3 \%$ better at distinguishing the various emulsion oil slicks from the ambient sea surface compared to the simulated HP features, while the best simulated HP features were $0.8 \%$ better than the FP features to distinguish the PO from the open water region. The best HP features show lower separability than the best FP features in the end of the $\sim 8$ hour time series compared to the beginning for the emulsion slicks. The FP features containing the cross-polarization scattering component are found to be best at distinguishing the various slicks from open water, 
Table VI: The mean and standard deviation of the JM from the best FP (top) and the best HP (bottom) feature along the time series for the various oil slicks and the open water regions.

\begin{tabular}{|c|c|c|c|c|}
\hline $\begin{array}{l}\text { Time } \\
\text { (UTC) }\end{array}$ & PO vs. OW & E40 vs. OW & E60 vs. OW & E80 vs. OW \\
\hline $05: 32$ & $\begin{array}{l}\zeta_{V V}(1.2 \pm 0.1) \\
\zeta_{R V}(1.32 \pm 0.12)\end{array}$ & $\begin{array}{l}\lambda_{3} \quad(1.48 \pm 0.08) \\
\zeta_{R V} \quad(1.49 \pm 0.1)\end{array}$ & $\begin{array}{l}\lambda_{3} \quad(1.25 \pm 0.07) \\
\zeta_{R V}(1.25 \pm 0.09)\end{array}$ & $\begin{array}{ll}\zeta_{V V} & (0.93 \pm 0.1) \\
\zeta_{R R} & (1.15 \pm 0.15) \\
\end{array}$ \\
\hline 05:46 & $\begin{array}{l}\lambda_{3}(1.01 \pm 0.11) \\
\operatorname{det}\left(C_{(R H, R V)}\right)(0.98 \pm 0.1)\end{array}$ & $\begin{array}{l}\lambda_{3}(1.21 \pm 0.07) \\
\operatorname{det}\left(C_{(R H, R V)}\right)(1.15 \pm 0.07)\end{array}$ & $\begin{array}{ll}\zeta_{X} \quad(1.2 \pm 0.04) \\
\zeta_{R R} \quad(1.13 \pm 0.07)\end{array}$ & $\begin{array}{l}\operatorname{det}\left(C_{(F P)}\right)(1.33 \pm 0.07) \\
\operatorname{det}\left(C_{(R R, R L)}\right)(1.4 \pm 0.11)\end{array}$ \\
\hline $06: 26$ & $\begin{array}{ll}\zeta_{V V} & (0.86 \pm 0.06) \\
\zeta_{R V} & (0.93 \pm 0.1)\end{array}$ & $\begin{array}{ll}\lambda_{3} & (0.88 \pm 0.07) \\
q_{1} & (0.94 \pm 0.1)\end{array}$ & $\begin{array}{ll}\lambda_{3} & (0.98 \pm 0.06) \\
q_{1} & (0.95 \pm 0.07)\end{array}$ & $\begin{array}{l}\lambda_{3}(0.92 \pm 0.07) \\
\zeta_{R R} \quad(0.92 \pm 0.08)\end{array}$ \\
\hline $06: 39$ & $\begin{array}{ll}\lambda_{3} & (0.8 \pm 0.1) \\
q_{1} & (0.79 \pm 0.1) \\
\end{array}$ & $\begin{array}{ll}\zeta_{X} & (0.68 \pm 0.08) \\
q_{1} & (0.61 \pm 0.09)\end{array}$ & $\begin{array}{ll}\zeta_{V V} & (0.84 \pm 0.06) \\
\zeta_{R R} & (0.83 \pm 0.08)\end{array}$ & $\begin{array}{l}\lambda_{3}(0.84 \pm 0.09) \\
\operatorname{det}\left(C_{(R R, R L)}\right)(0.82 \pm 0.12)\end{array}$ \\
\hline 07:05 & $\begin{array}{l}\operatorname{det}\left(C_{(F P)}\right)(0.93 \pm 0.12) \\
\zeta_{R R}(0.92 \pm 0.14)\end{array}$ & $\begin{array}{l}\lambda_{3}(0.86 \pm 0.12) \\
\operatorname{det}\left(C_{(R H, R V)}\right)(0.71 \pm 0.12)\end{array}$ & $\begin{array}{l}\lambda_{3}(1 \pm 0.12) \\
\operatorname{det}\left(C_{(R R, R L)}\right)(0.91 \pm 0.12)\end{array}$ & $\begin{array}{l}\lambda_{3} \quad(1.01 \pm 0.1) \\
\zeta_{R R} \quad(0.96 \pm 0.11)\end{array}$ \\
\hline 07:17 & $\begin{array}{ll}i_{C O} & (0.99 \pm 0.08) \\
\zeta_{R R} & (1.14 \pm 0.07) \\
\end{array}$ & $\begin{array}{ll}\zeta_{X} & (0.64 \pm 0.13) \\
q_{1} & (0.67 \pm 0.09) \\
\end{array}$ & $\begin{array}{ll}\lambda_{3} & (0.68 \pm 0.13) \\
q_{1} & (0.64 \pm 0.13)\end{array}$ & $\begin{array}{ll}\lambda_{3} & (0.85 \pm 0.12) \\
q_{1} & (0.83 \pm 0.14)\end{array}$ \\
\hline 07:31 & $\begin{array}{ll}\zeta_{X} & (0.81 \pm 0.06) \\
q_{1} & (0.82 \pm 0.13) \\
\end{array}$ & $\begin{array}{l}\lambda_{3}(0.89 \pm 0.07) \\
\operatorname{det}\left(C_{(R H, R V)}\right)(0.75 \pm 0.11)\end{array}$ & $\begin{array}{ll}\lambda_{3} & (0.7 \pm 0.07) \\
q_{1} & (0.66 \pm 0.08)\end{array}$ & $\begin{array}{l}\zeta_{X}(0.89 \pm 0.04) \\
\operatorname{det}\left(C_{(R H, R V)}\right)(0.82 \pm 0.09)\end{array}$ \\
\hline $07: 44$ & $\begin{array}{l}\zeta_{X}(0.84 \pm 0.06) \\
\zeta_{R R}(0.93 \pm 0.13)\end{array}$ & $\begin{array}{ll}\zeta_{X} & (0.67 \pm 0.06) \\
q_{1} & (0.69 \pm 0.09)\end{array}$ & $\begin{array}{ll}\zeta_{X} & (0.75 \pm 0.05) \\
q_{1} & (0.76 \pm 0.1)\end{array}$ & $\begin{array}{ll}\zeta_{X} & (0.87 \pm 0.05) \\
q_{1} & (0.87 \pm 0.04)\end{array}$ \\
\hline 07:57 & $\begin{array}{l}\lambda_{3}(1.18 \pm 0.06) \\
\operatorname{det}\left(C_{(R R, R L)}\right)(1.02 \pm 0.1)\end{array}$ & $\begin{array}{l}\lambda_{3} \quad(0.83 \pm 0.06) \\
\zeta_{R R} \quad(0.77 \pm 0.1)\end{array}$ & $\begin{array}{l}\zeta_{V V} \quad(0.72 \pm 0.06) \\
q_{1}(0.69 \pm 0.08)\end{array}$ & $\begin{array}{l}\lambda_{3} \quad(1.01 \pm 0.05) \\
\zeta_{R R} \quad(0.95 \pm 0.1)\end{array}$ \\
\hline 08:11 & $\begin{array}{ll}\zeta_{V V}(0.92 \pm 0.1) \\
\zeta_{R R}(1.08 \pm 0.09)\end{array}$ & $\begin{array}{l}\lambda_{3} \quad(0.84 \pm 0.12) \\
\zeta_{R R}(0.84 \pm 0.12)\end{array}$ & $\begin{array}{ll}\lambda_{3} & (0.96 \pm 0.11) \\
q_{1} & (0.93 \pm 0.12)\end{array}$ & $\begin{array}{l}\lambda_{3} \quad(1.04 \pm 0.1) \\
\zeta_{R V}(1.04 \pm 0.1)\end{array}$ \\
\hline $08: 24$ & $\begin{array}{ll}\lambda_{3} & (0.98 \pm 0.06) \\
q_{1} & (0.88 \pm 0.09)\end{array}$ & $\begin{array}{ll}\lambda_{3} & (0.71 \pm 0.06) \\
q_{1} & (0.61 \pm 0.08)\end{array}$ & $\begin{array}{l}\zeta_{V V}(0.79 \pm 0.06) \\
q_{1}(0.77 \pm 0.07)\end{array}$ & $\begin{array}{ll}\zeta_{V V} & (0.85 \pm 0.05) \\
\zeta_{R V} & (0.92 \pm 0.08)\end{array}$ \\
\hline 08:37 & $\begin{array}{ll}\zeta_{X} & (1.05 \pm 0.08) \\
q_{0} & (1.04 \pm 0.15)\end{array}$ & $\begin{array}{l}\lambda_{3} \quad(0.81 \pm 0.09) \\
\zeta_{R V} \quad(0.89 \pm 0.16)\end{array}$ & $\begin{array}{l}\lambda_{3} \quad(0.85 \pm 0.09) \\
\zeta_{R V} \quad(0.84 \pm 0.09)\end{array}$ & $\begin{array}{ll}\lambda_{3} & (0.97 \pm 0.07) \\
q_{1} & (0.94 \pm 0.07)\end{array}$ \\
\hline $11: 45$ & $\begin{array}{ll}\zeta_{V V} & (1.21 \pm 0.08) \\
\zeta_{R R} & (1.42 \pm 0.13)\end{array}$ & $\begin{array}{lr}\zeta_{X} & (0.68 \pm 0.08) \\
\zeta_{R R} & (0.7 \pm 0.16)\end{array}$ & $\begin{array}{ll}\zeta_{X} & (0.88 \pm 0.07) \\
\zeta_{R R} & (0.94 \pm 0.12)\end{array}$ & $\begin{array}{l}\lambda_{3} \quad(0.97 \pm 0.08) \\
\zeta_{R R}(0.98 \pm 0.14)\end{array}$ \\
\hline $12: 00$ & $\begin{array}{ll}\zeta_{X} & (0.85 \pm 0.06) \\
q_{1} & (0.83 \pm 0.08)\end{array}$ & $\begin{array}{ll}\lambda_{3} & (0.55 \pm 0.07) \\
q_{1} & (0.41 \pm 0.1)\end{array}$ & $\begin{array}{l}\lambda_{3} \quad(0.53 \pm 0.06) \\
\zeta_{R V} \quad(0.46 \pm 0.11)\end{array}$ & $\begin{array}{ll}\zeta_{X} & (0.48 \pm 0.07) \\
\zeta_{R V} & (0.5 \pm 0.1)\end{array}$ \\
\hline $12: 14$ & $\begin{array}{ll}\zeta_{X} & (1.04 \pm 0.05) \\
\zeta_{R V} & (1.18 \pm 0.07)\end{array}$ & $\begin{array}{ll}\zeta_{X} & (0.48 \pm 0.05) \\
\zeta_{R V} & (0.48 \pm 0.09)\end{array}$ & $\begin{array}{ll}\zeta_{X} & (0.8 \pm 0.06) \\
\zeta_{R R} & (0.83 \pm 0.06)\end{array}$ & $\begin{array}{ll}\lambda_{3} & (0.93 \pm 0.06) \\
q_{1} & (0.92 \pm 0.08) \\
\end{array}$ \\
\hline $12: 29$ & $\begin{array}{ll}\lambda_{3} & (1.02 \pm 0.07) \\
q_{1} & (0.89 \pm 0.1) \\
\end{array}$ & $\begin{array}{ll}\lambda_{3} & (0.6 \pm 0.08) \\
q_{1} & (0.56 \pm 0.11) \\
\end{array}$ & $\begin{array}{l}\lambda_{3}(0.69 \pm 0.08) \\
\operatorname{det}\left(C_{(R R, R L)}\right)(0.63 \pm 0.06)\end{array}$ & $\begin{array}{l}\lambda_{3}(0.8 \pm 0.08) \\
\operatorname{det}\left(C_{(R H, R V)}\right)(0.81 \pm 0.1)\end{array}$ \\
\hline 13:03 & $\begin{array}{l}\zeta_{X} \quad(1.09 \pm 0.07) \\
\zeta_{R V} \quad(1.15 \pm 0.15)\end{array}$ & $\begin{array}{l}\zeta_{X} \quad(0.42 \pm 0.09) \\
\zeta_{R V} \quad(0.39 \pm 0.11)\end{array}$ & $\begin{array}{l}\zeta_{X} \quad(0.66 \pm 0.09) \\
\zeta_{R V}(0.61 \pm 0.14)\end{array}$ & $\begin{array}{ll}\lambda_{3} & (0.8 \pm 0.09) \\
q_{1} & (0.7 \pm 0.15) \\
\end{array}$ \\
\hline $13: 18$ & $\begin{array}{l}\lambda_{3}(1.28 \pm 0.06) \\
\operatorname{det}\left(C_{(R H, R V)}\right)(1 \pm 0.09)\end{array}$ & $\begin{array}{l}\mu_{C} \quad(0.78 \pm 0.32) \\
\phi_{(R H, R V)} \quad(0.31 \pm 0.36)\end{array}$ & $\begin{array}{ll}\lambda_{3} & (0.64 \pm 0.07) \\
q_{1} & (0.54 \pm 0.08)\end{array}$ & $\begin{array}{ll}\lambda_{3} & (0.53 \pm 0.07) \\
q_{1} & (0.51 \pm 0.03)\end{array}$ \\
\hline
\end{tabular}

however these cross-polarization features are not possible to isolate when using the HP mode. The $\zeta_{R R}, \zeta_{R V}, q_{1}$, and $q_{0}$ are good alternatives to separate the slicks from the open water regions using the HP mode. High separability values between the oil slicks and open water were also obtained using the $\operatorname{det}\left(\boldsymbol{C}_{(R H, R V)}\right)$ and $\operatorname{det}\left(\boldsymbol{C}_{(R R, R L)}\right)$, and their potential should be further investigated for other types of oil under various wind and ocean conditions. Overall, the best FP features are $\zeta_{X}, \operatorname{det}\left(\boldsymbol{C}_{(F P)}\right), \lambda_{3}$, and $\zeta_{V V}$.

This study reveals a high correspondence between the results and the scattering theory of the two-scale Bragg model. All the features that showed poor detectability of the oil slicks are independent of the ocean wave spectrum (the small-scale roughness), while the features resulting in good separability were dependent, amongst other factor, on the ocean wave spectrum.

This study highlights the importance of performing an incidence angle correction on the complex scattering vector prior to segmentation.

In general, the plant oil has the highest detectability across the full time series for both the FP and the HP modes, and its detectability does not decrease at the end of the UAVSAR time series, as is the case for the emulsion slicks. It was not possible to discriminate the plant oil from the emulsion slicks, which might be a result of the high wind and the relatively small volume of the released oils.

Our findings suggest that similar slick-sea separability performance can be achieved using either HP or FP data at high wind conditions and for small slicks in volume. However, this study should be repeated for data collected in other wind conditions and for various oil thicknesses. Further investigation should be conducted to determine whether real HP data could achieve the same results as both the FP and simulated HP data. Real HP data would reduce complexity (compared to the FP mode) of the sensor in terms of average power, on-board mass, data volume, and provide more design flexibility.

\section{ACKNOWLEDGMENT}

The authors would like to thank NOFO for hosting NORSE2015 and MET Norway for collecting the meteorological and ocean observations. This research was carried out in part at the Jet Propulsion Laboratory, California Institute of Technology, under contract with the National Aeronautics and Space Administration. NORSE2015 was partly financed by CIRFA (RCN Grant no. 237906). This work is supported by the NORRUSS program (RCN Grant no. 233896). UAVSAR data is courtesy of NASA/JPL Caltech. The authors will also 
like to thank the reviewers for their useful comments and suggestions.

\section{APPENDIX A \\ INCIDENCE ANGLE CORRECTION}

The UAVSAR instrument images at incidence angles between 20 and 65 degrees [5], and the ocean backscatter is known to decrease with increasing incidence angle. The oil slick regions in the UAVSAR time series are selected based on a segmentation method that is discussed in Section IV-C The intensity variation related to incidence angle can be larger than the intensity difference between the classes, and hence the oil slicks might be neglected in the original segmentation. Furthermore, the oil slicks spread out in the range direction with time, increasing the incidence angle span across the slicks. Hence, the effects from the incidence angle on the output regions are more significant in the last passes of the UAVSAR time series when the slicks have spread out. Therefore, to avoid the incidence angle effect dominating the segmentation, and to allow incidence angle independent comparison across the time series, an incidence angle correction is performed on the scattering vector prior to multilooking, segmentation, and feature computation. The incidence angle correction is obtained from the following expression [69]:

$$
\boldsymbol{S}(\theta)=\boldsymbol{S}^{\prime} \sqrt{\frac{\sin (\theta)}{\sin \left(\theta_{r e f}\right)}} \Longrightarrow \boldsymbol{S}^{\prime}=\boldsymbol{S}(\theta) \sqrt{\frac{1}{\gamma(\theta)}},
$$

where $\boldsymbol{S}(\theta)$ is the measured scattering vector dependent on the incidence angle, $\theta$ is the incidence angle, $\theta_{\text {ref }}$ is the reference angle, $S^{\prime}$ is the corrected scattering vector independent of the incidence angle, and $\gamma(\theta)$ is the $\frac{\sin (\theta)}{\sin \left(\theta_{r e f}\right)}$ fraction. Because we are dealing with the complex scattering vector, rather than intensities, the square root is applied. Range and incidence angle are related in a one-to-one correspondence, hence the $\gamma(r)$ rather than $\gamma(\theta)$ is used.

To preserve the polarimetry in the data, the same $\gamma(r)$ should be used when correcting the different complex scattering components, i.e., $\mathbf{S}_{H H}, \mathbf{S}_{H V}$, and $\mathbf{S}_{V V}$. Selecting different $\gamma(r)$ for each complex scattering component could influence the various multivariate polarimetric features, like the determinant of the covariance matrix. Rather than determining the relation between range and incidence angle, we estimate $\gamma(r)$ empirically from the span of the intensities ( $\operatorname{span}=$ $\left.I_{H H}+I_{V V}+I_{H V}\right)$ by considering a region of clean water (no ships nor oil slicks), and assuming that this region is homogenous and has no texture. For this work, the region along the range direction was selected from the span, and contained 1000 pixels in the azimuth direction. An intensity profile $\left(I_{R g}^{\mathrm{span}}\right)$ from the span was created by taking the average of that region, and these values were further smoothed in the range direction. The reference level was chosen to be the mean value of the total power along the range direction $\left(\hat{I}_{R g}^{\mathrm{span}}\right) \cdot \gamma(r)$ can be estimated as:

$$
\gamma(r) \approx \frac{I_{R g}^{\mathrm{span}}}{\hat{I}_{R g}^{\mathrm{span}}}
$$

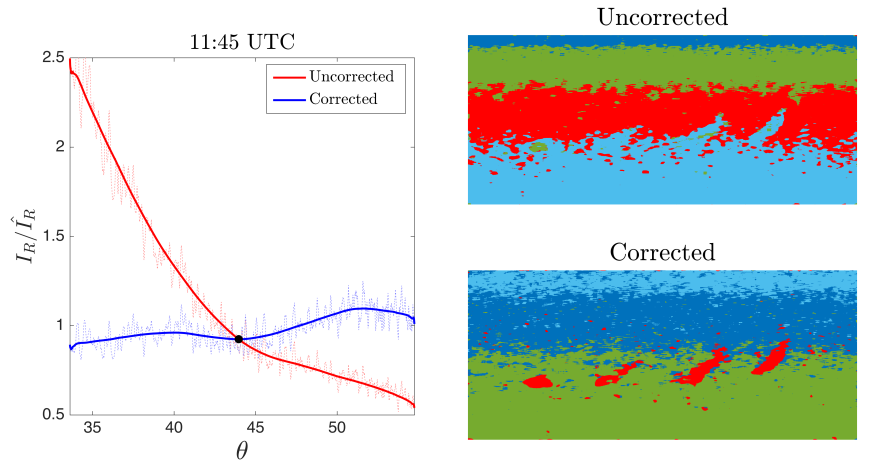

Figure 12: Illustration of the incidence angle correction applied to the UAVSAR scene acquired at 11:45 UTC. The left figure shows the smoothed mean VV-intensity profiles normalized to the mean of the span profile using the clean sea region before (red line) and after correction (blue line). The dotted lines are the unsmoothed mean intensity profiles. The black marker represents the reference level, i.e., mean of the span along the range direction. The right figures show the results of the segmentation with and without incidence angle correction. The colors represent the various output segments.

Fig. 12 illustrates the incidence angle correction applied to an ascending UAVSAR scene. The blue line is the corrected smoothed mean VV-intensity along the range direction (normalized to the mean intensity value), and the dashed blue line is the unsmoothed corrected mean intensity value along the range direction, also normalized. Here, we use the incidence angle (covering the location of the oil slicks) on the $\mathrm{x}$-axes. The red line is the smoothed uncorrected mean VV-intensity profile, and the dashed red line is the unsmoothed version of that profile. Only the VV-intensity is used to demonstrate this, but the same behavior was observed for the $\mathrm{HH}$ and HV intensities. After applying this correction method, the incidence angle dependence of the scattering components is reduced.

The corrected intensity profile is not a perfectly flat curve in any of the individual channels, which might be because they are corrected based on span, and the visible polarimetric variation indicates that there is some local variations.

From visual inspection of the right panel in Fig. 12, it is clear that the intensities are significantly affected by the incidence angle. One example of how the incidence angle effects the segmentation results are also illustrated in Fig. 12 . Here, the upper-right figure displays the results of the segmentation using uncorrected data as input, while the lowerright image is when the incidence angle correction is applied prior to the segmentation method. Clearly, the segmentation method did not successfully locate the oil slicks in the uncorrected case. However, if corrected data is used the segmentation algorithm successfully identifies the oil slicks. These segmentation results highlight how important it is to perform the incidence angle correction prior to segmentation. This correction is done on all the UAVSAR scenes prior to segmentation and feature computation.

Calculating the intensity values from the corrected scattering 
vector yields an approximation of the damping ratio. This is because the entire intensity image is normalized using the mean of a chosen open water region $\left(I_{R g}^{\mathrm{span}}\right)$. The corrected intensities are named damping ratios (see Table III and $\mathrm{V}$, and are labeled $\zeta$, for example:

$$
\zeta_{V V}=\left\langle\left|S_{V V}^{\prime}\right|^{2}\right\rangle
$$

where $\langle\cdot\rangle$ represents the averaging (multilooking).

\section{REFERENCES}

[1] B. Minchew, C. E. Jones, and B. Holt, "Polarimetric Analysis of Backscatter From the Deepwater Horizon Oil Spill Using L-Band Synthetic Aperture Radar," IEEE Trans. Geosci. Remote Sens., vol. 50, no. 10, pp. 3812-3830, Oct. 2012.

[2] S. Skrunes, C. Brekke, and T. Eltoft, "Characterization of Marine Surface Slicks by Radarsat-2 Multipolarization Features," IEEE Trans. Geosci. Remote Sens., vol. 52, no. 9, pp. 5302-5319, Sep. 2014.

[3] S. Skrunes, C. Brekke, T. Eltoft, and V. Kudryavtsev, "Comparing NearCoincident C- and X-Band SAR Acquisitions of Marine Oil Spills," IEEE Trans. Geosci. Remote Sens., vol. 53, no. 4, pp. 1958-1975, Apr. 2015.

[4] M. Migliaccio, F. Nunziata, and A. Gambardella, "On the co-polarized phase difference for oil spill observation," Int. J. Remote Sens., vol. 30, no. 6, pp. 1589-1602, Mar. 2009.

[5] A. G. Fore, B. D. Chapman, B. P. Hawkins, S. Hensley, C. E. Jones, T. R. Michel, and R. J. Muellerschoen, "UAVSAR Polarimetric Calibration," IEEE Trans. Geosci. Remote Sens., vol. 53, no. 6, pp. 3481-3491, Jun. 2015.

[6] R. K. Raney, "Hybrid-Polarity SAR Architecture," IEEE Trans. Geosci. Remote Sens., vol. 45, no. 11, pp. 3397-3404, Nov. 2007.

[7] F. J. Charbonneau, B. Brisco, R. K. Raney, H. McNairn, C. Liu, P. W. Vachon, J. Shang, R. DeAbreu, C. Champagne, A. Merzouki, and T. Geldsetzer, "Compact Polarimetry Overview and Applications Assessment," Can. J. Remote Sens., vol. 36, no. 2, pp. 298-315, 2010.

[8] J.-C. Souyris, P. Imboa, R. Fjørtoft, S. Mingot, and J.-S. Lee, "Compact Polarimetry Based on Symmetry Properties of Geophysical Media: The

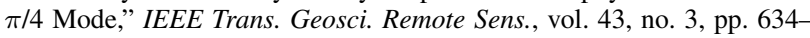
646, Mar. 2005.

[9] F. Nunziata, M. Migliaccio, and X. Li, "Sea Oil Slick Observaion Using Hybrid-Polarity SAR Architecture," IEEE J. Ocean. Eng., vol. 40, no. 2, pp. 426-440, 2015.

[10] A.-B. Salberg, O. Rudjord, and A. H. S. Solberg, "Oil Spill Detection in Hybrid-Polarimetric SAR Images," IEEE Trans. Geosci. Remote Sens., vol. 52, no. 10, pp. 6521-6533, Oct. 2014.

[11] M. J. Collins, M. Denbina, B. Minchew, C. E. Jones, and B. Holt, "On the Use of Simulated Airborn Compact Polarimetric SAR for Characterizing Oil-Water Mixing of the Deepwater Horizon Oil Spill," IEEE J. of Sel. Topics Appl. Earth Obs. Remote Sen., vol. 8, no. 3, pp. 1062-1077, Mar. 2015.

[12] R. Shirvany, M. Chabert, and J.-Y. Tourneret, "Ship and Oil-Spill Detection using the Degree of Polarization in Linear and Hybrid/Compact Dual-Pol SAR," IEEE J. of Sel. Topics Appl. Earth Obs. Remote Sen., vol. 5, no. 3, pp. 885-892, Jun. 2012.

[13] B. Minchew, "Determining the mixing of oil and sea water using polarimetric synthetic aperture radar," Geophys. Res. Lett., vol. 39, no. $16,2012$.

[14] W. Alpers and H. Hühnerfuss, "Radar Signatures of Oil Films Floating on the Sea Surface and the Marangoni Effect," J. of Geophys. Res., vol. 93, no. C4, pp. 3642-3648, Apr. 1988.

[15] G. R. Valenzuela, "Theories for the interaction of electromagnetic and oceanic waves - A review," Boundary-Layer Meteorology, vol. 12, no. 1-4, pp. 61-85, Jan. 1978.

[16] B. Holt, "SAR imaging of the ocean surface," Synthetic Aperture Radar Marine User's Manual, no. 25-80, C. Jackson and J. Apel, Eds. U.S. Depratment of Commerce, National Oceanic and Atmospheric Administration, C. Jackson and J. Apel, 2004.

[17] R. Romeiser and W. Alpers, "An improved composite surface model for the radar backscattering cross section of the ocean surface 1. theory of the model and optimization/validation by scatterometer data," $J$. of Geophys. Res., vol. 102, no. C11, pp. 25 237-25 250, Nov. 1997.

[18] C. Brekke, C. Jones, S. Skrunes, B. Holt, M. Espeseth, and T. Eltoft, "Cross-Correlation Between Polarization Channels in SAR Imagery Over Oceanographic Features," IEEE Geosci. Remote Sens. Lett., vol. 13, no. 7, pp. 997-1001, Jul. 2016.
[19] S. Skrunes, C. Brekke, C. E. Jones, and B. Holt, "A Multisensor Comparison of Experimental Oil Spills in Polarimetric SAR for High Wind Conditions," IEEE J. of Sel. Topics Appl. Earth Obs. Remote Sen., vol. 9, no. 11, pp. 4948-4961, Jun. 2016.

[20] C. E. Jones, K.-F. Dagestad, Ø. Breivik, B. Holt, J. Röhrs, K. H. Christensen, M. Espeseth, C. Brekke, and S. Skrunes, "Measurement and Modeling of Oil Slick Transport," J. Geophys. Res. Oceans, vol. 121, no. 10, pp. 2169-9291, 2016.

[21] M. E. Nord, T. L. Ainsworth, J.-S. Lee, and N. Stacy, "Comparison of Compact Polarimetric Synthetic Aperture Radar Modes," IEEE Trans. Geosci. Remote Sens., vol. 47, no. 1, pp. 634-646, Mar. 2009.

[22] S. R. Cloude, Polarisation Applications in Remote Sensing, First, Ed. Oxford University Press, 2010.

[23] R. K. Raney, "A Perspective on Compact Polarimetry," IEEE Geosci. and Rem. Sensing Newsletters, Sept. 2011.

[24] M. J. Collins, M. Denbina, and G. Atteia, "On the Reconstruction of Quad-Pol SAR Data from Compact Polarimetry Data for Ocean Target Detection," IEEE Trans. Geosci. Remote Sens., vol. 51, no. 1, pp. 591600, Jan. 2013.

[25] R. K. Raney, "Comparing Compact and Quadrature Polarimetric SAR Performance," IEEE Geosci. Remote Sens. Lett., vol. 13, no. 6, pp. 861864, Jun. 2016.

[26] A. Iodice, A. Natale, and D. Riccio, "Retrieval of soil surface parameters via a polarimetric two-scale model," IEEE Trans. Geosci. Remote Sens., vol. 49, no. 7, pp. 2531-2547, Jul. 2011.

[27] J.-S. Lee, D. L. Schuler, and T. L. Ainsworth, "Polarimetric SAR Data Compensation for Terrain Azimuth Slope Variation," IEEE Trans. Geosci. Remote Sens., vol. 38, no. 5, pp. 2153-2163, Sep. 2000.

[28] M. M. Espeseth, S. Skrunes, C. Brekke, A.-B. Salberg, C. E. Jones, and B. Holt, "Oil spill characterization in the hybrid polarity SAR domain using log-cumulants," Proc. SPIE, Image and Signal Processing for Remote Sensing XXII, vol. 10004, no. 14, Oct. 2016.

[29] C. E. Jones, M. M. Espeseth, B. Holt, C. Brekke, and S. Skrunes, "Characterization and discrimination of evolving mineral and plant oil slicks based on L-band synthetic aperture radar (SAR)," Proc. SPIE, SAR Image Analysis, Modeling, and Techniques XVI, vol. 10003, Oct. 2016.

[30] M. Basseville, "Distance measures for signal processing and pattern recognition," Signal Processing, vol. 18, no. 4, pp. 349-369, 1989.

[31] Y. Li, H. Lin, Y. Zhang, and J. Chen, "Comparisons of Circular Transmit and Linear Receive Compact Polarimetric SAR Features for Oil Slicks Discrimination," Journal of Sensors, vol. 2015, p. Article ID 631561, 2015.

[32] M. Migliaccio, A. Gambardella, and M. Tranfaglia, "SAR Polarimetry to Observe Oil Spills," IEEE Trans. Geosci. Remote Sens., vol. 45, no. 2, pp. 506-511, Feb. 2007.

[33] M. Dabbor, S. Howell, M. Shokr, and J. Yackel, "The Jeffries-Matusita distance for the case of complex Wishart distribution as a separability criterion for fully polarimetric SAR data," Int. J. Remote Sens., vol. 35, no. 19 , pp. 6859-6873, Oct. 2014.

[34] P. H. Swain and S. M. Davis, "Remote sensing: The quantitative approach," New York: McGraw-Hill, 1978.

[35] M. Dabboor and T. Geldsetzer, "On the Classification of Sea Ice Types using Simulated Radarsat Constellation Mission (RCM) Compact Polarimetric SAR Parameters," ASPRS 2014 Annual Conference, Louisville, Kentucky, Mar. 2014.

[36] M. Migliaccio, M. Tranfaglia, and S. A. Ermakov, "A Physical Approach for the Observation of Oil Spills in SAR images," IEEE J. Ocean. Eng., vol. 30, no. 3, pp. 496-507, Jul. 2005.

[37] F. Galland, P. Réfrégier, and O. Germain, "Synthetic Aperture Radar Oil Spill Segmentation by Stochastic Complexity Minimization," IEEE Geosci. Remote Sens. Lett., vol. 1, no. 4, pp. 295-299, Oct. 2014.

[38] S. Derrode and G. Mercier, "Unsupervised multiscale oil slick segmentation from SAR images using a vector HMC model," Pattern Recognition, vol. 40, no. 3, pp. 1135-1147, Mar. 2007.

[39] A. P. Doulgeris and T. Eltoft, "Scale mixture of Gaussian modelling of polarimetric SAR data,," EURASIP Journal on Advances in Signal Processing, no. 8, Jan. 2010.

[40] A. P. Doulgeris, "A simple and extendable segmentation method for multi-polarisation SAR images," POLINSAR, Frascati, 2013.

[41] V. Wismann, M. Gade, W. Alpers, and H. Huhnerfuss, "Radar signatures of marine mineral oil spills measured by an airborne multi-frequency radar," Int. J. Remote Sens., vol. 19, no. 18, pp. 3607-3623, 1998.

[42] N. Pinel, C. Bourlier, and I. Sergievskaya, "Two-dimensional radar backscattering modeling of oil slicks at sea based on the model of local balance: Validation of two asymptotic techniques for thick films," IEEE Trans. Geosci. Remote Sens., vol. 52, no. 5, pp. 2326-2338, May 2014. 
[43] D. L. Schuler, J. S. Lee, and K. W. Hoppel, "Polarimetric SAR image signatures of the ocean and Gulf Stream features," IEEE Trans. Geosci. Remote Sens., vol. 31, no. 6, pp. 1210-1221, Nov. 1993.

[44] D. Velotto, M. Migliaccio, F. Nunziata, and S. Lehner, "Dual-Polarized TerraSAR-X Data for Oil-Spill Observation," IEEE Trans. Geosci. Remote Sens., vol. 49, no. 12, pp. 4751-4762, Dec. 2011.

[45] W. Wenguang, L. Fei, W. Peng, and W. Jun, "Oil spill detection from polarimetric SAR image," ICSP2010 Proceedings, 2010.

[46] B. Zhang, W. Perrie, X. Li, and W. G. Pichel, "Mapping sea surface oil slicks using RADARSAT-2 quad-polarization SAR image," Geophys. Res. Lett., vol. 38, no. 10, May 2011.

[47] V. Kudryavtsev, B. Chapron, A. Myasoedova, F. Collard, and J. Johannessen, "On dual co-polarized SAR measurements of the ocean surface," IEEE Geosci. Remote Sens. Lett., vol. 10, no. 4, pp. 761-765, Jul. 2013.

[48] W. Tian, Y. Shao, J. Yuan, S. Wang, and Y. Liu, "An experiment for oil spill recognition using RADARSAT-2 image," 2010 IEEE Int. Geosci. Remote Sens. Symp., pp. 2761-2764, Jul. 2010.

[49] M. Migliaccioa, F. Nunziata, and A. Buono, "SAR polarimetry for sea oil slick observation," Int. J. Remote Sens., vol. 36, no. 12, pp. $3243-$ 3273, Jun. 2015.

[50] D. L. Schuler and J.-S. Lee, "Mapping ocean surface features using biogenic slick-fields and SAR polarimetric decomposition techniques," IEE Proc. Radar, Sonar and Navigation, vol. 153, no. 3, pp. 260-270, Jun. 2006

[51] M. Migliaccio, A. Gambardella, F. Nunziata, M. Shimada, and O. Isoguchi, "The PALSAR polarimetric mode for sea oil slick observation," IEEE Trans. Geosci. Remote Sens., vol. 47, no. 12, pp. 4032 4041, Dec. 2009.

[52] F. T. Ulaby, K. Sarabandi, and A. Nashashibi, "Statistical properties of the Muller matrix of distributed targets," IEE Proceedings F - Radar and Signal Processing, vol. 139, no. 2, pp. 136-146, Apr. 1992.

[53] M. R. Drinkwater, R. Kwok, E. Rignot, H. Israelsson, R. G. Onstott, and D. P. Winebrenner, "Chapter 24. Potential Applications of Polarimetry to the Classification of Sea Ice," Microwave Remote Sensing of Sea Ice, Geophysical Monograph 68, American Geophysical Union, 1992.

[54] J.-S. Lee and E. Pottier, Polarimetric Radar Imaging, From Basics to Applications, B. J. Thompson, Ed. Boca Raton, FL, USA: CRC Press Taylor and Francis Group, 2009.

[55] W. Taylor and M. Boerner, "Basic Concepts in Radar Polarimetry," PolSARpro Lecture Notes, vol. 3, 2007.

[56] H. Li, W. Perrie, Y. Zhou, and Y. He, "Oil spill detection on the ocean surface using hybrid polarimetric SAR imagery," Science China, vol. 59, no. 2, pp. 249-257, Feb. 2016

[57] H. Li, E. P. Y. He, J. Wu, and X. Luo, "Analysis of the Polarimetric SAR Scattering Properties of Oil-Covered Waters," IEEE J. of Sel. Topics Appl. Earth Obs. Remote Sen., vol. 8, no. 8, pp. 3751-3759, Aug. 2015.

[58] S. Cloude, D. Goodenough, and H. Chen, "Compact Decomposition Theory," IEEE Trans. Geosci. Remote Sens., vol. 9, no. 1, pp. 28-32, Jan. 2012.

[59] K. Raney, J. T. S. Cahill, G. W. Patterson, and D. B. J. Bussey, "The m-chi Decomposition of Hybrid Dual-Polarimetric Radar Data with Application to Lunar Craters," J. of Geophys. Res., vol. 117, no. E12, May 2012.

[60] B. Slade, "Radarsat-2 product description," MacDonald, Dettwiler and Associates Ltd., Rickmond, BC, USA, RN-SP-52-1238, 2011.

[61] T. Fritz and M. Eineder, "TerraSAR-X Ground Segment Basic Product Specification Document," Oct. 2010.

[62] D. Latini, F. D. Frate, and C. E. Jones, "Multi-frequency and polarimetric quantitative analysis of the gulf of mexico oil spill event comparing different SAR systems systems," Remote Sens. Env., vol. 183, no. 183, pp. 26-42, Sep. 2016

[63] T. Misra, S. S. Rana, N. M. Desai, D. B. Dave, Rajeevjyoti, R. K. Arora, C. V. N. Rao, B. V. Bakori, R. Neelakantan, and J. G. Vachchani, "Synthetic Aperture Radar payload on-board RISAT-1: configuration, technology and performance," Current science, vol. 104, no. 4, Feb. 2013.

[64] S. Skrunes, C. E. Jones, C. Brekke, B. Holt, and M. M. Espeseth, "On the Effect of Imaging Geometry on Multipolarization SAR Features for Oil Spill Observation,” Proc. Living Planet Symp., 2016.

[65] R. Shirvany, M. Chabert, and J.-Y. Tourneret, "Estimation of the Degree of Polarization for Hybrid/Compact and Linear Dual-Pol SAR Intensity Images: Principles and Applications," IEEE Trans. Geosci. Remote Sens., vol. 51, no. 1, pp. 539-551, Jan. 2013.

[66] A. H. S. Solberg, "Remote Sensing of Ocean Oil-Spill Pollution," Proceedings of the IEEE, vol. 100, no. 10, pp. 2931-2945, Oct. 2012.

[67] J. Coleman, Oil in the Sea III. Washington DC, USA: National Academy Press, 2003.
[68] W. Alpers and H. A. Espedal, "Oils and surfactants," Synthetic Aperture Radar Marine User's Manual, C. R. Jackson and J. R. Ape, Eds. Washington DC, USA: U.S. Department of Commerce, National Oceanic and Atmosphereic Administration, pp. 263-275, Sep. 2004.

[69] G. Sun, K. J. Ranson, and V. I. Kharuk, "Radiometric slope correction for forest biomass estimation from SAR data in Western Sayani Mountains, Siberia," Remote Sens. Env., vol. 79, no. 2-3, pp. 279-287, 2002.

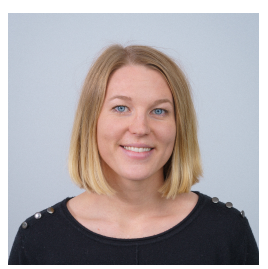

Martine M. Espeseth received the M.Sc. degree from the Department of Physics and Technology, UiT The Arctic University of Norway, Troms $\varnothing$, Norway, in 2015

She is currently a Ph.D. student at the Centre for Integrated Remote Sensing and Forecasting for Arctic Operations (CIRFA) at UiT The Arctic University of Norway. From February 2016 to April 2016, she was a Visiting Ph.D. student with the Jet Propulsion Laboratory, California Institute of Technology, Pasadena, CA, USA. Her current research interests include remote sensing of polarimetric SAR and with a focus on compact polarimetry within both marine oil pollution and sea ice applications.

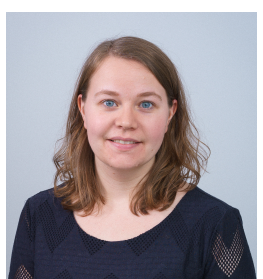

Stine Skrunes (S'12-M'15) received the M.Sc. and $\mathrm{Ph} . \mathrm{D}$. degrees from the Department of Physics and Technology, UiT The Arctic University of Norway, Tromsø, Norway, in 2011 and 2014, respectively.

She is currently a Postdoc at the same department, with the Centre for Integrated Remote Sensing and Forecasting for Arctic Operations (CIRFA). Her current research interests include remote sensing of ocean areas, specifically by polarimetric SAR and with a focus on marine oil pollution.

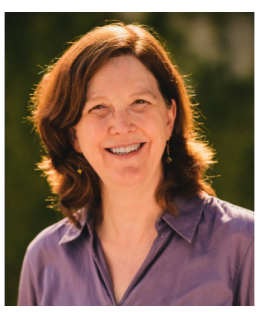

Cathleen E. Jones (M'94) is a radar scientist at NASA's Jet Propulsion Laboratory, California Institute of Technology, where her main research is focused on using radar remote sensing for studying natural disasters and monitoring critical infrastructure, primarily using high resolution L-band PolSAR and InSAR based on UAVSAR data. Her research includes development of methods for determining oil slick characteristics and identifying levee deformation, seepage, and general subsidence rates using SAR. In addition she has done work detecting sinkhole precursor in InSAR-challenged areas. She received a B.S. in physics from Texas A\&M and a Ph.D. in physics from the California Institute of Technology. 


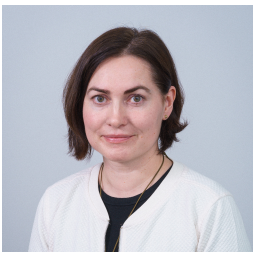

Camilla Brekke (M'12) received the Cand.Sci. and $\mathrm{Ph} . \mathrm{D}$. degrees from the University of Oslo, Oslo, Norway, in 2001 and 2008, respectively.

In 2001, she was a System Developer with Ericsson, Asker, Norway. From 2001 to 2002, she was a Young Graduate Trainee with the European Space Agency, Noordwijk, The Netherlands. From 2002 to 2009, she was a Scientist with the Norwegian Defense Research Establishment (FFI), Kjeller, Norway. In 2009, she joined the Department of Physics and Technology, UiT The Arctic University of Norway, Troms $\varnothing$, Norway, as an Associate Professor. From October 2012 to January 2013, she was a Visiting Scientist with the Jet Propulsion Laboratory, California Institute of Technology, Pasadena, CA, USA. She is currently with the Earth Observation Laboratory and the Centre for Integrated Remote Sensing and Forecasting for Arctic Operations, UiT. Her current research interests cover signal and image processing of synthetic aperture radar data for arctic and marine applications.

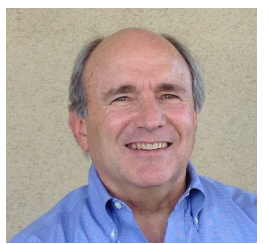

Benjamin Holt (M'88) is a research scientist in the Ocean Circulation group within the Earth Science Section at the Jet Propulsion Laboratory, California Institute of Technology, which he joined in 1978.

He received the B.S. from Stanford University in 1972 and the M.S. in physical oceanography from the University of Southern California in 1988. His research interests include using multisensor remote sensing data to examine the geophysical state of polar sea ice and snow, coastal oceanography and circulation, and the detection of marine pollutants. In addition, he is also involved with new instrument development and techniques for microwave measurement of sea ice thickness.

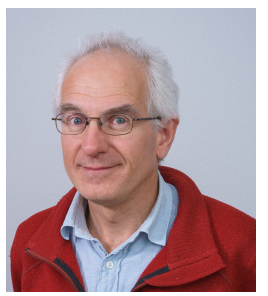

Anthony P. Doulgeris (S'06-M'12) received the B.Sc. degree in physics from The Australian National University, Canberra, Australia, in 1988, the M.Sc. degree and the Ph.D. degree in physics from the Department of Physics and Technology, UiT The Arctic University of Norway, Tromsø, Norway, in 2006, and 2011, respectively.

He joined the Department of Physics and Technology at UiT The Arctic University of Norway, in 2007 and is currently an associate professor in applied Earth observation. His research interests focus on investigating remote sensing, pattern recognition and multidimensional statistical modeling, in particular with polarimetric synthetic aperture radar images. 